\title{
Phase changes of the Be/X-ray binary X Persei
}

\author{
J. S. Clark ${ }^{1,2}$, A. E. Tarasov ${ }^{3}$, A. T. Okazaki ${ }^{4,5}$, P. Roche ${ }^{6}$, and V. M. Lyuty ${ }^{7}$ \\ 1 Department of Physics and Astronomy, University College London, Gower Street, London, WC1E 6BT, UK \\ 2 Astronomy Centre, CPES, Univ. of Sussex, Falmer, Brighton BN1 9QH, UK \\ 3 Crimean Astrophysical Observatory and Isaac Newton Institute of Chile, Crimean Branch, Naucny, \\ 98409 Crimea, Ukraine \\ 4 Faculty of Engineering, Hokkai-Gakuen University, Toyohira-ku, Sapporo 062-8605, Japan \\ 5 Institute of Astronomy, Madingley Road, Cambridge CB3 0HA, UK \\ 6 Department of Physics \& Astronomy, University of Leicester Leicester LE1 9RH, UK \\ 7 Sternberg Astronomical Institute, Universiteskii pr. 13, 119899 Moscow, Russia
}

Received 8 August 2001 / Accepted 18 October 2001

\begin{abstract}
We present high resolution optical spectroscopy and $V$ band photometry obtained during the period 1987-2001 for the Be star X Persei/HD 24534, the counterpart to the X-ray pulsar 4U 0352+30. We find that throughout this interval X Per is highly active, with significant photometric and spectroscopic variability. We identify one episode of complete disc loss during this period (1988 May-1989 June), characterised by significant $\Delta V=0.6 \mathrm{mag}$ optical fading and the presence of purely photospheric $\mathrm{H} \alpha$ and He I $6678 \AA$ lines. Two further episodes of pronounced optical fading which did not result in the complete dispersal of the circumstellar disc were also identified (1994 October-1995 October and 1999 November-present). The emission line profiles of both H $\alpha$ and He $6678 \AA$ also show significant variability. Cyclic changes in the strength of the peaks in both emission lines are observed, with periods ranging from 0.6-2 yrs - we attribute these to the presence of a one armed density wave in the inner circumstellar disc. Additional structure at large projected velocities is also present in the He I line - suggesting the presence of a significant density enhancement in the disc near the stellar surface (the "double disc" of Tarasov \& Roche). The evolution of the outer edge of the $\mathrm{H} \alpha$ emitting region of the circumstellar disc is followed during disc formation, and is found to increase rather slowly. This observation, combined with the presence of the one armed density wave and the rate of disc formation and loss all provide strong evidence for the hypothesis that the circumstellar disc of $\mathrm{X}$ Per is a viscous decretion disc, with angular momentum being supplied by an as yet unknown physical mechanism near the stellar surface.
\end{abstract}

Key words. circumstellar matter - stars: early type - stars: individual: X Persei $-4 \mathrm{U} 0352+30$

\section{Introduction}

$\mathrm{X}$ Persei/HD 24534 is the prototypical Be/X-ray binary system, a subset of High Mass X-ray binaries consisting of an early-type non-supergiant $\mathrm{Oe} / \mathrm{Be}$ star and a compact, evolved companion. Oe/Be stars are characterised by the presence of emission lines in their spectra (typically $\mathrm{HI}$, He I and low excitation metals such as Fe II) and a pronounced IR continuum excess due to free-free/free-bound $(\mathrm{ff} / \mathrm{fb})$ emission, both originating in a gaseous, equatorially concentrated circumstellar disc around the OB star. Given that the circumstellar disc acts as a reservoir of material for the compact object to accrete from, detailed knowledge of the long term behaviour of the circumstellar environment of the Be star is crucial to understanding the $\mathrm{X}$-ray properties of $\mathrm{Be} / \mathrm{X}$-ray binaries.

Send offprint requests to: J. S. Clark, e-mail: jsc@star.ucl.ac.uk
X Per is classified as B0Ve (Lyubimkov et al. 1997, henceforth L97; Roche et al. 1997, henceforth R97) ${ }^{1}$. Recently Delgado-Marti et al. (2001) determined an orbital period of 250 days and eccentricity of 0.11 , implying an inclination of $\sim 23^{\circ}-30^{\circ}$ from RXTE data. X Per has been observed to undergo considerable spectroscopic and photometric variability in recent decades (Roche et al. 1993, henceforth R93; Norton et al. 1991). Photometric and spectroscopic variability is a relatively common feature of Be stars - indeed Dougherty \& Taylor (1994) find that $18 \%$ of a sample of 125 field Be stars are variable at near-IR wavelengths. This is interpreted as being due to changes in the physical properties of the circumstellar disc surrounding the Be star, such as density and/or geometry. A small subset of variable Be stars show far more

\footnotetext{
${ }^{1}$ Table 1 presents the main results from the spectral and luminosity classification of L97, which was omitted from published paper, where Table 4 is reproduced twice in place of Table 5.
} 
Table 1. Mass, radius, luminosity and age of X Per estimated from evolutionary models of single stars (correct version of Table 5 from L97).

\begin{tabular}{cccc}
\hline $\log M / M_{\odot}$ & $\log R / R_{\odot}$ & $\log L / L_{\odot}$ & $t\left(10^{6}\right.$ years $)$ \\
\hline $1.19 \pm 0.11$ & $0.81 \pm 0.15$ & $4.45 \pm 0.39$ & $5.3 \pm 2.1$ \\
\hline
\end{tabular}

extreme behaviour, characterised by large $(\sim 1 \mathrm{mag})$ photometric variability accompanied by dramatic changes in emission line profiles - in some cases varying from pure emission to photospheric profiles and vice versa. These are thought to be due to global changes in the circumstellar environment of the Be star, such as the complete loss or reformation of the circumstellar disc - referred to as phase changes. Given that there is at present no accepted physical mechanism for the formation, support (and loss) of the equatorial discs around Be stars such rare events are of considerable interest, providing unique insights into the Be star phenomenon.

In this paper we present high resolution optical spectroscopy and $V$ band photometry of X Per over the period 1987 to 2000 , encompassing the $\mathrm{Be} \rightarrow \mathrm{B} \rightarrow$ Be phase changes between 1987 July-1994 May and subsequent disc activity. The paper is ordered as follows. Section 2 summarises the spectroscopic and photometric history of $\mathrm{X}$ Per, while in Sect. 3 we present the optical spectroscopy and photometry. Section 4 discusses the observed variability in the context of phase changes in other Be stars, the implications of the behaviour of X Per for the viscous decretion disc model for Be stars (Lee et al. 1991; see also Porter 1999; Okazaki 2001) and the interaction between the circumstellar envelope and the neutron star companion. Finally, we present our conclusions in Sect. 5, and summarise the observational data in Appendix A.

\section{Previous photometric and spectroscopic behaviour: 1913-1982}

Given the variability shown by a significant minority of Be stars it it is important to place our current observations of X Per in the context of its long term spectroscopic and photometric history. These observations, dating from the turn of last century (e.g. X Per has been observed as part of the Univ. of Michigan study of bright Be stars since 1913) suggest that throughout this period X Per has been highly active, with significant spectroscopic and photometric variability.

Cowley et al. (1972) report that between 1913-1971 the Balmer lines were strongly variable - weak in the period 1913-41, becoming stronger towards 1946 before fading again by 1947, then strengthening to a maximum by 1962 and decreasing thereafter. Sporadic He I (4472, 4922 and $5015 \AA$ ) and weak He II (4686 $\AA$ ) emission is present during this time. Brucato \& Kristian (1972) also note that Fleming (1912) had reported P Cygni Balmer lines profiles "near the turn of the century" - corresponding to a period of unusual photometric activity (Muller \& Kempf 1907) - which differ from the double peaked line profiles typically reported for $\mathrm{X}$ Per.

Significant variations in the Violet $(V)$ and Red $(R)$ peak ratios of the Balmer and Fe II emission lines were noted by Mc Laughlin in 1929, 1934 and 1936 (1937; Cowley et al. 1972), a pattern which continued between November 1957-January 1961, when Wackerling (1972) describes abrupt changes in the $V / R$ ratio of the Balmer and metallic emission lines - the ratio changing from $\sim 1.5$ in Feb. 1957 to $\sim 0.6$ by the autumn of that year. Systematic, cyclic variability was again observed in late 1959-early 1960 with the $V / R$ ratio plot of $\mathrm{H} \beta$ shown in Cowley et al. (1972) clearly showing the onset of cyclic $V / R$ variability around 1960 , with a possible period of around 10 years.

Following this period of activity in the 1960's X Per again displayed spectroscopic variability around the time of it's discovery as an X-ray source in 1970. By 1971 clear P Cygni Balmer line profiles were again observed (Brucato \& Kristian 1972), suggesting mass outflow from the system. Observations by Galkina (1977, 1980, 1983, 1986a, 1986b) cover the time interval from 1974 to 1982 (JD 2442369-2445326), when the longterm lightcurve of $\mathrm{X}$ Per displayed strong variability, including an extended low photometric state in 1974-1977 (although $\mathrm{H} \alpha$ is still in emission during this period), further low states of shorter duration in 1979 and 1981 and high-luminosity phases in 1978 and 1980 (R93). Cyclic variability in $\mathrm{H} \alpha$ was again observed between 1974-75 (Galkina 1980).

From these observations two distinct forms of variability appear to have been present in X Per throughout the last century. The first is a cyclic variability in the peak ratio of the emission lines of H I, He I and metallic lines with periods varying between $\sim 1-10 \mathrm{yrs}$, the second a long term fluctuation in the optical brightness $(V \sim 6.7-6.1)$, accompanied by changes in the strength of emission lines. These forms of variability have been observed in other Be stars and have respectively been interpreted as the presence of a prograde one armed oscillation in the circumstellar disc (e.g. Okazaki 1997), and as the phase changes briefly described in Sect. 1 (these findings will be further discussed in the light of our new observations in Sect. 4).

\section{Optical spectroscopic and photometric data}

The observations presented in this paper come from a number of different sources and are summarised in Appendix A, giving details of the source of the spectra and basic parameters. The source of each spectrum is listed, and corresponds to:

cr - Crimean Astrophysical Observatory, Ukraine, $2.6 \mathrm{~m}$, Coudé focus, with GEC (pre-1995) or EEV (1995 onwards) CCD, resolving power 25000;

uk - either Isaac Newton Telescope, INT, La Palma, 2.5 m, Cassegrain focus, Intermediate Dispersion Spectrograph (IDS), various CCDs, low resolution or JKT, Jakobus Kapteyn Telescope, La Palma, 1 m, Cassegrain focus, 
Richardson Brealey Spectrograph (RBS) various CCDs, low resolution;

cz - Ondrejov Observatory, Czech Republic, 2 m, Coudé focus, Reticon 1800, resolving power $\sim 20000$ (Vojtech Simon; priv. comm.);

fr - Observatoire de Haute Provence, France, $1.5 \mathrm{~m}$, Aurelie echelle spectrograph, EEV-CCD, resolving power 80000 .

Finally the $V$ band photometric observations presented in Figs. 7 and 8 were obtained with a pulse-counting photometer (EMI 9789 tube) attached to the $0.6 \mathrm{~m}$ telescope of the Crimean Laboratory of Sternberg Astronomical Institute, Nauchny, Ukraine (in addition to those data already presented by R97).

The reduced spectra are presented in Figs. 1-3 (H $\alpha)$ and Figs. 4-6 (He I 6678 $\AA$ ) - note that the low resolution spectra are not shown - while we summarise the observational properties of the lines in Figs. 7 and 8. In both Figs. 7 and 8 panel $a$ presents contemporaneous $V$ band photometry while panel $b$ presents the $E W$ of the emission line in question. Panels $c$ and $d$ of Fig. 7 presents the evolution of the peak to peak ratio and peak separation for $\mathrm{H} \alpha$ where both parameters have been measured by fitting (non-physical) Gaussian profiles to the data. Panels $c$ and $d$ of Fig. 8 present the peak position and separation for He I $6678 \AA$, again measured by fitting Gaussians to the emission line profiles. We emphasise that this procedure was simply adopted to standardise the measurement procedure; it should not be interpreted as a physical model for the observations.

\subsection{Summary of $H \alpha$ and $H e I 6678 \AA$ line variability}

In order to discuss the behaviour of these lines in relation to each other, and to the overall photometric behaviour of $\mathrm{X}$ Per we have divided the $V$ band lightcurve into distinct regions, which we have called phases 1 to 4 , as indicated in Figs. 7 and 8. These divisions were adopted after examination of the lightcurves and spectra and were chosen to mark significant changes in the properties of the data (and therefore the inferred properties of the circumstellar envelope).

- Phase 1 (TJD 46500-48100; Fig. 1):

Covers the initial photometric bright state and subsequent fading ( $\Delta V \sim 0.6$ mags. in $\sim 400$ days) between TJD $\sim$ 47300-47700, when X Per enters into an extended low photometric state (ELS).

$\mathbf{H} \boldsymbol{\alpha}$ : Phase 1 is poorly covered spectroscopically (and no He I $6678 \AA$ observations exit for this phase), but our data shows that prior to TJD 47237 the $\mathrm{H} \alpha$ line is bright $(E W \sim-10 \AA)$, with a double-peaked structure and apparent $V / R$ peak ratio variability. The peak separation at this time is rather small $\left(\sim 140 \mathrm{~km} \mathrm{~s}^{-1}\right)$. After TJD 47237 the $E W$ of $\mathrm{H} \alpha$ is also observed to decrease dramatically, the end of this phase has been chosen to coincide with the last time $\mathrm{H} \alpha$ is observed in emission.

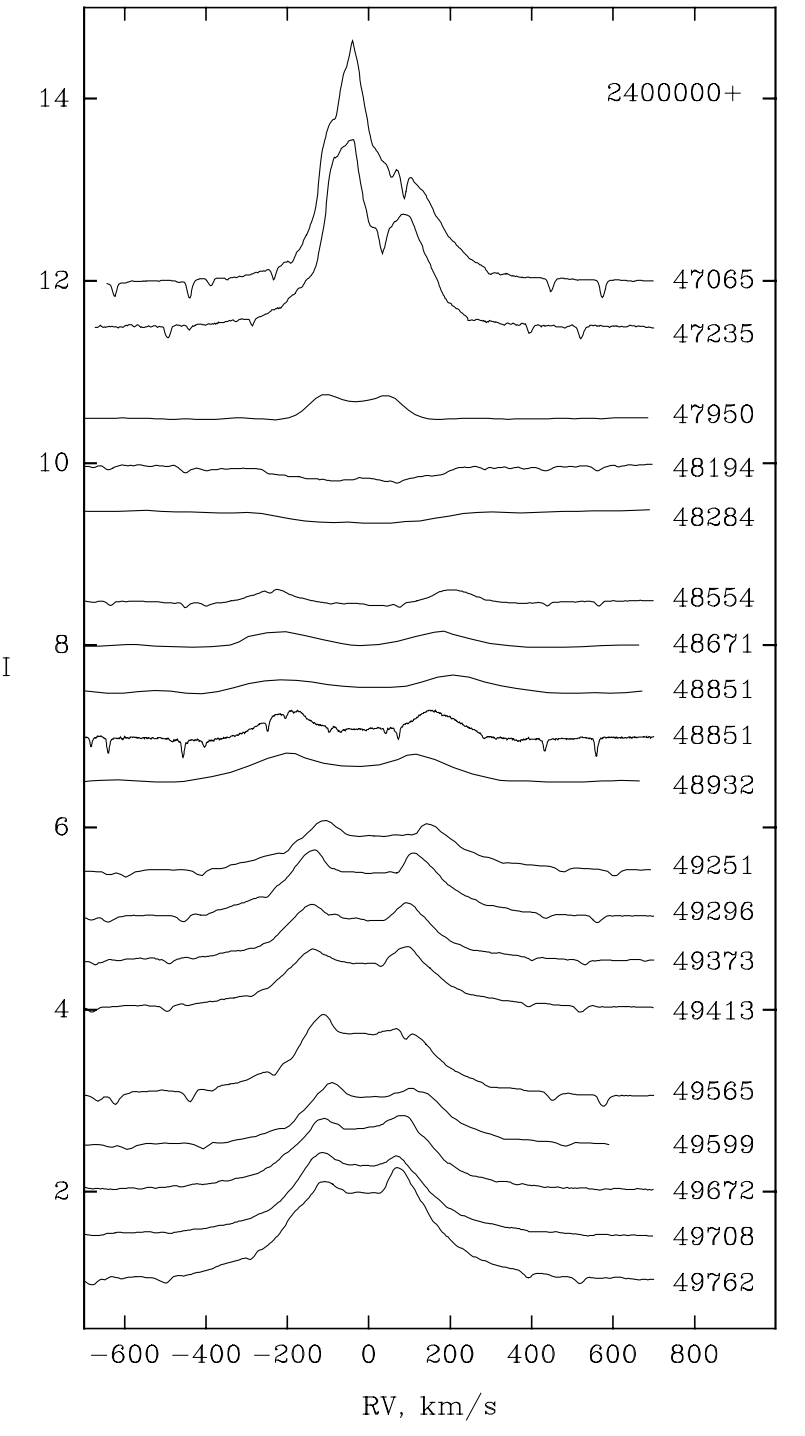

Fig. 1. Normalised $\mathrm{H} \alpha$ spectra of X Per covering the period 1987 Sep.-1995 Feb. Axes are velocity relative to line centre and line intensity normalised to the stellar continuum.

- Phase 2 (TJD 48100-49937; Figs. 1, 2, 4): Between TJD 48100-49100 X Per remains in the ELS, with very low amplitude photometric variations (Percy 1992; R97), possibly increasing in size towards end of this period. Between TJD 49100-49650 the ELS ends with rapid brightening in the $V$ band (0.6 mag in $\sim 550$ days), peaking at $V \sim 6.23$ on TJD 49630. Unlike Phase 1 no plateau of $\sim$ constant brightness is observed and the light curve starts to decay on a similar timescale to that observed in Phase 1 ( $\Delta V \sim 0.5 \mathrm{mag}$ in $\sim 470$ days; also see Figs. 7 and 8 ), although it does not reach the extreme observed in the ELS.

$\mathbf{H} \boldsymbol{\alpha}$ : Phase 2 was defined to begin with occurence of the first detection of the photospheric profile of $\mathrm{H} \alpha$, indicating complete disc loss at this time (note that this occurs significantly later than the commencement of the photometric ELS). This state persists for only a short time before emission is again detectable, with the line having moved back into net emission by TJD 48554 (Figs. 1 and 7, 


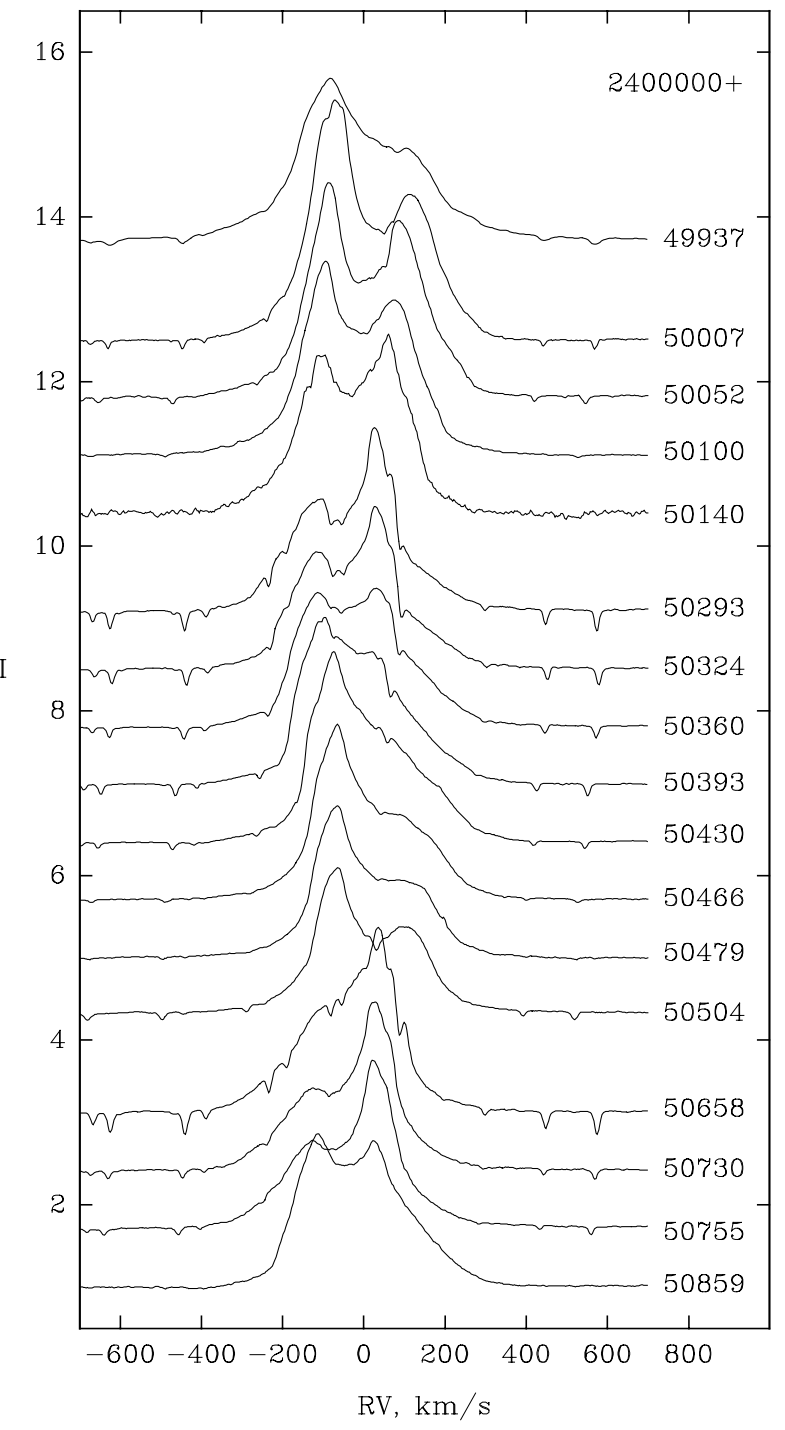

Fig. 2. Normalised $\mathrm{H} \alpha$ spectra of $\mathrm{X}$ Per covering the period 1995 Aug. -1998 Feb. Axes are the same as Fig. 1.

Table A.1). Initially detectable as a weak double peaked line profile with a large peak separation $\left(\sim 500 \mathrm{~km} \mathrm{~s}^{-1}\right)$ the intensity and $E W$ of the line increases linearly with a corresponding decrease in peak separation, until TJD 49565 when the decrease ends and low level $V / R$ variability is observed (although a periodicity cannot be identified at this time).

He I 6678 A: initially in absorption the line gradually moves into emission (first observed on TJD 48851) with widely separated peaks and a central (photospheric) absorption component. As with $\mathrm{H} \alpha$, between TJD 4885149700 the peak separation decreases monotonically with the underlying photospheric profile becoming visible in the line wings by TJD $\sim 49600$ (Fig. 4). The $E W$ variability is more pronounced than for $\mathrm{H} \alpha$ at this time, reaching a local minimum when star is at maximum brightness (TJD 49630). By TJD 49708 there is infilling in the photospheric wings of the line and by TJD 49762 the line has a pronounced 4 peaked profile as reported by

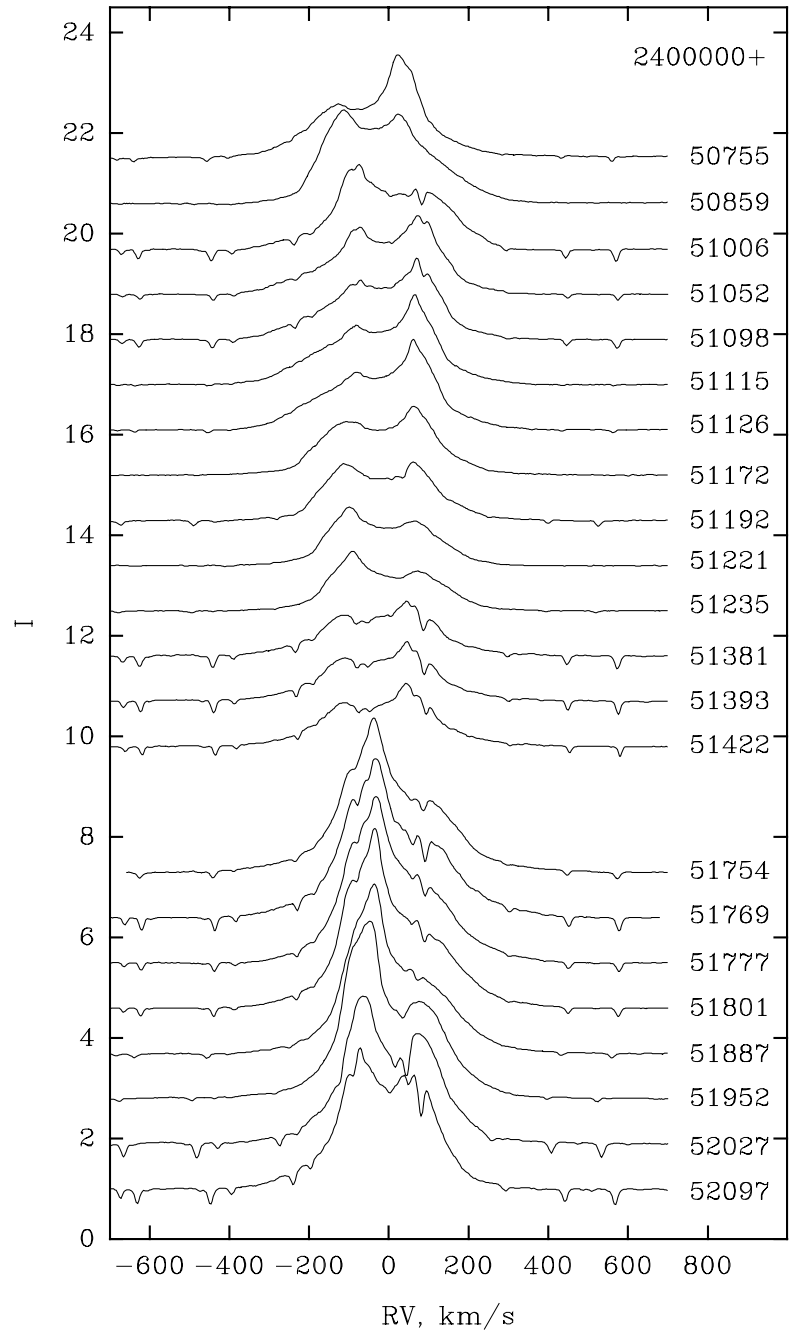

Fig. 3. Normalised $\mathrm{H} \alpha$ spectra of $\mathrm{X}$ Per covering the period 1998 Feb.-2000 Sep. Axes are the same as Fig. 1.

Tarasov \& Roche (1995). Subsequently, as X Per fades in the $V$ band the $E W$ of the line increases to $\sim-1.1 \AA$ by the end of Phase 2 .

- Phase 3 (TJD 49937-51052; Figs. 2-5):

Phase 3 represents a prolonged period ( $\sim 3$ yrs) of low level $(\Delta V \sim 0.2 \mathrm{mag})$ photospheric fluctuations on timescales of $\sim 100$ days, ending with a dramatic brightening that signals the onset of Phase 4. Spectroscopic observations at this time reveal significant activity during this time, although the peak separation of the $\mathrm{H} \alpha$ line remains $\sim$ constant throughout this time (TJD 50293-51052).

$\mathbf{H} \boldsymbol{\alpha}$ : pronounced cyclical $V / R$ variability is seen throughout this period with three maxima observed. Both the amplitude of variations and cycle length appear variable during this period with $\tau_{\max } \rightarrow \min \sim 356,289$ and 117 days for the descending branches of the 3 cycles present in Phase 3 (the last cycle extending into Phase 4), implying variable cycle lengths of $\sim 2-0.6$ yrs respectively (see Fig. 7). Throughout this period the $E W$ shows small fluctuations of $\sim 2 \AA$ about a mean level of $\sim-10 \AA$ and the peak separation stabilises at $\sim 140 \mathrm{~km} \mathrm{~s}^{-1}$; the same 


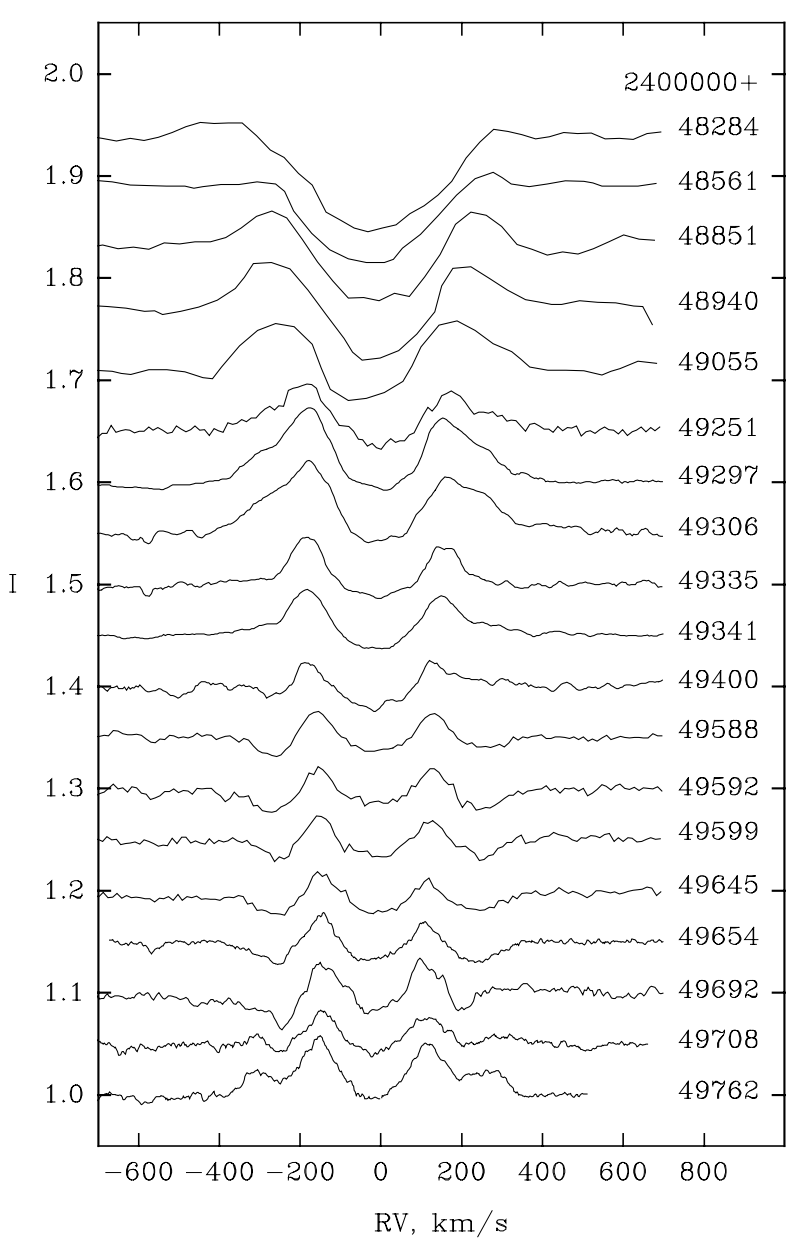

Fig. 4. Normalised He I $6678 \AA$ spectra of X Per covering the period 1991 Jan.-1995 Feb. Axes are the same as Fig. 1.

value observed in spectra obtained prior to the photometric ELS.

He I 6678 Å: during this phase HeI shows a complicated, multi-component structure, with strong variability in both intensity and radial velocity for all peaks. Although the characteristic 4 peaked profile observed at the end of Phase 2 has disappeared by the start of Phase 3, 182 days later, significant emission at large velocities is found to persist throughout Phase 3 with identifiable peaks in the line profile observed to migrate rapidly towards line centre (at a greater rate than the original peaks; Fig. 8). Initially the stronger central emission peaks are observed to vary in intensity in the same manner as $\mathrm{H} \alpha$ before the motion of the outer peaks causes them to become indistinguishable, forming a variable 3 peaked profile superimposed on a broad plateau of emission. Given the strength of the broad emission wings of $\mathrm{H} \alpha$ we suspect that similar high velocity emission components may also be present (Figs. 2 and 3) in this line as well - however we are unable to unambiguously deconvolve the line into its components. Given the rapid evolution of the He I line profile, the seasonal breaks in observations between TJD 50139-274, 50504-658 and 50755-859 prevent the detailed study of the evolution of individual emission peaks

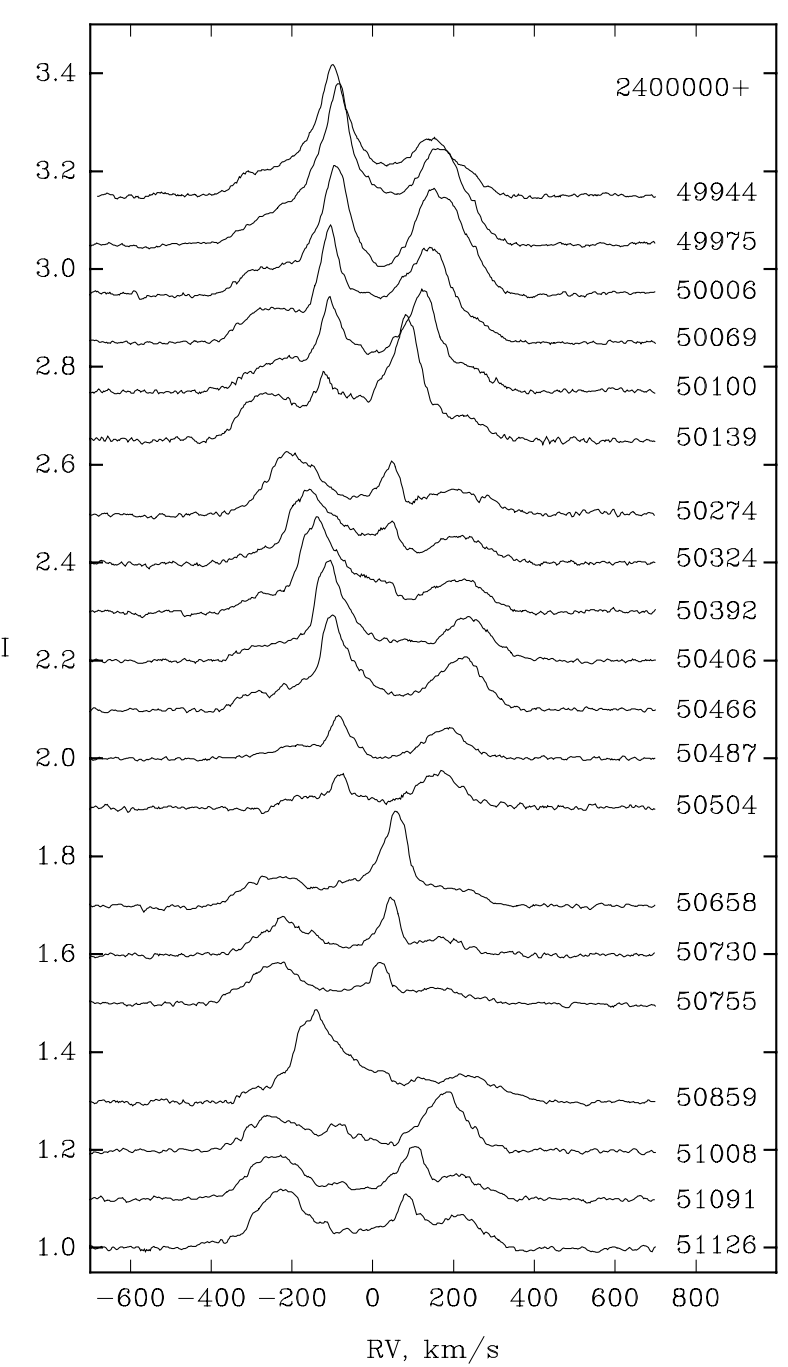

Fig. 5. Normalised He I $6678 \AA$ spectra of X Per covering the period 1995 Aug. -1998 Nov. Axes are the same as Fig. 1.

throughout Phase 3. Nevertheless, where temporal coverage is sufficient it appears that both peak strength and position vary systematically, suggesting that the cyclic $V / R$ variability visible in $\mathrm{H} \alpha$ is also present in He I $6678 \AA$, superimposed on a broad plinth of emission.

- Phase 4 (TJD 51052-present; Figs. 3, 5, 6): The final phase covers a second episode of large amplitude photometric variability, of a qualitatively different nature to that of Phase 2. Beginning around TJD 51050 the $V$ band abruptly brightens by $\sim 0.5$ mag in only 190 days, compared to $\sim 400$ day rise in Phase 2. A plateau lasting $\sim 1$ yr is then observed followed by an apparent slow decline in brightness in comparison to the abrupt, rapid dimming following the photometric maximum in Phase 2.

$\mathbf{H} \boldsymbol{\alpha}$ : the cyclic $V / R$ variability is observed to continue throughout Phase 4 with a highly variable amplitude and apparent periodicity $\left(\tau_{\max } \rightarrow \min \sim 201\right.$ days for the descending branch of the first cycle and the data are too sparse to accurately determine the period of the second cycle). The $E W$ of the line is also highly variable, reaching a peak value of $\sim-17 \AA$ some 400 days after the peak 


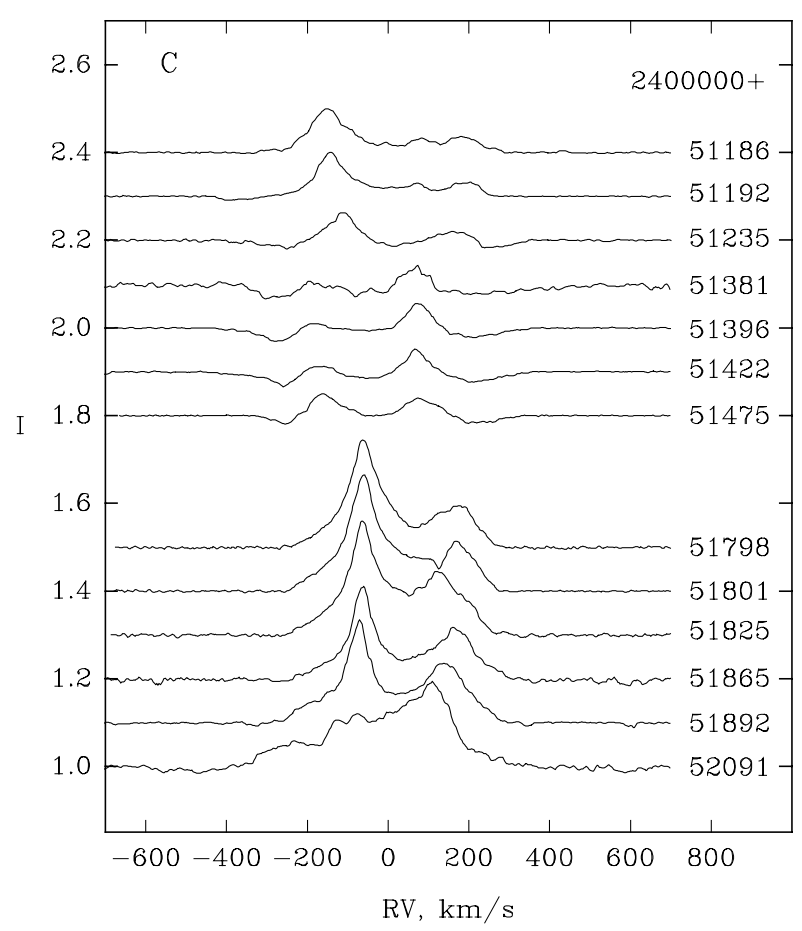

Fig. 6. Normalised He I $6678 \AA$ spectra of X Per covering the period 1999 Jan.-2000 Dec. Axes are the same as Fig. 1.

in the $V$ band photometry. A similar lag was also observed in Phases 2 and 3 and will be further discussed in Sect. 4.

He I $6678 \AA$ A: as with $\mathrm{H} \alpha$ during this phase He I continues to show significant variability (possibly associated with the $V / R$ variability observed in the $\mathrm{H} \alpha$ profile at this time), with a noticeable reduction in line strength between TJD $\sim 51052-51475$. During this period emission at large velocities is observed to decay, revealing the wings of the underlying photospheric profile by TJD 51475. Subsequent observations $\sim 320$ days later reveal a significant strengthening of the line, with new emission visible at high velocities again obscuring the photospheric profile. This behaviour, seen during a period in which the photometry indicates the star is fading, mirrors that seen during the similar photometric fade between TJD 49600-50000.

\section{Discussion}

As can be seen X Per is highly variable throughout the period 1987-2001, with both the cyclic line variability and the phase changes between $\mathrm{Be} \rightarrow \mathrm{B} \rightarrow$ Be identified in the archival data (Sect. 2) present during this time. Given that both cyclic $V / R$ variability and phase changes appear ubiquitous for Be stars it is useful to examine these data in the light of the prevailing theoretical explanations for these behaviours.

Of particular importance to the following discussion is the presence of the neutron star companion in a long $\left(P_{\text {orb }}=250\right.$ days $)$, modestly eccentric $(e=$ 0.11 ) orbit (derived from pulse timing analyses of $R X T E$ observations; Delgado-Marti et al. 2001). The orbital

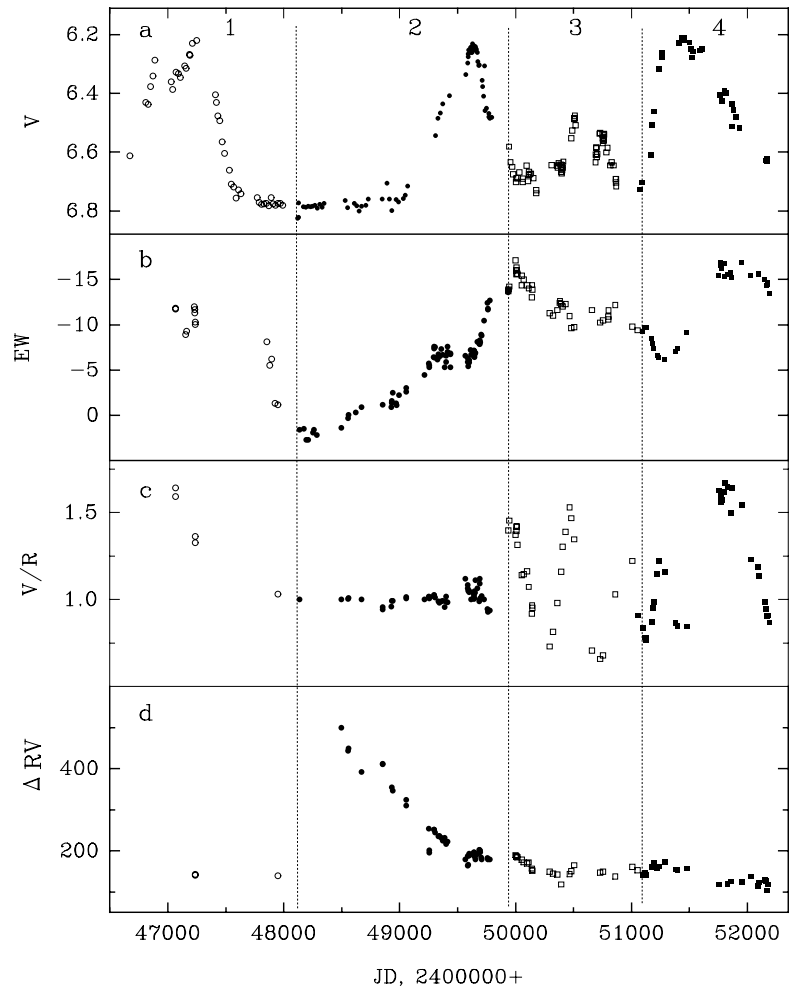

Fig. 7. Time variability of the main parameters of the $\mathrm{H} \alpha$ line. Panel a) displays the $V$ band lightcurve, b) displays the Equivalent Width (in $\AA$ ), c) the $V / R$ peak intensity variability and $\mathbf{d}$ ) the peak separation (in $\mathrm{km} \mathrm{s}^{-1}$ ). Note the symbols used reflect the phases we define in Sect. 3.1 - open circles for Phase 1, filled circles for Phase 2, open squares for Phase 3 and filled squares for Phase 4.

analysis of Delgado-Marti et al. (2001) further allowed the determination of the orbital separation, $a_{x} \sin i=454 \mathrm{lt}-\mathrm{s}$, and assuming a canonical mass of $1.4 M_{\odot}$ for the neutron star companion constrains the orbital inclination to $\sim 23^{\circ}$ $30^{\circ}$ (implying an orbital separation of $60-77 R_{*}$ ). This also allows us to constrain the rotational velocity of the Be star - the projected rotational velocity of $215 \pm 10 \mathrm{~km} \mathrm{~s}^{-1}$ (Lyubimkov 1997) translates to an absolute velocity of $430-550 \mathrm{~km} \mathrm{~s}^{-1}$, or $81-105 \%$ of the breakup velocity for $\mathrm{X}$ Per - implying that if the orbital and equatorial planes are coincident the inclination of the system must lie at the higher end of the range given by Delgado-Marti et al. (2001).

\subsection{Disc loss and reformation}

Disc loss \& reformation has been observed for both isolated (e.g. $\mu$ Cen; Hanuschik et al. 1993) and binary Be stars (e.g. V635 Cas $=4 \mathrm{U} 0115+63$, Negueruela et al. 2001) although the precise mechanism governing this behaviour and the support of the circumstellar disc is uncertain. Based on spectroscopic observations of $\mu$ Cen Hanuschik et al. (1993) suggest that discrete mass ejections from the star - which are subsequently circularised in orbit - are responsible for disc formation; 


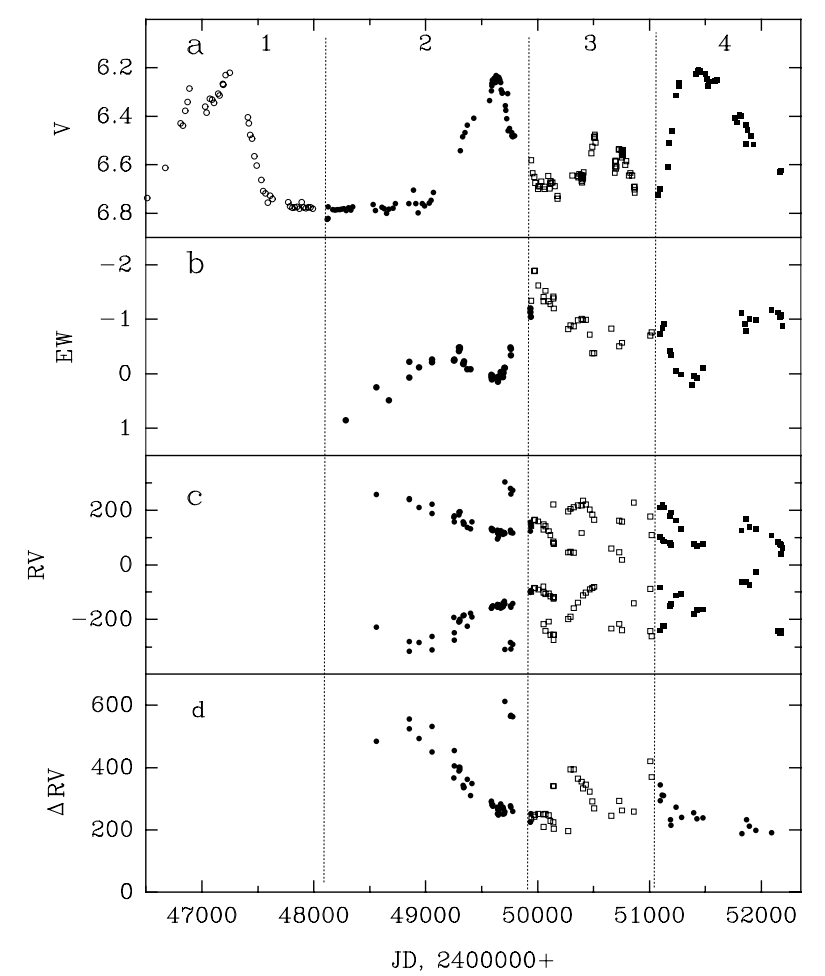

Fig. 8. Time variability of the main parameters of the HeI 6678 line. Panel a) displays the $V$ band lightcurve, Panel b) displays the Equivalent Width (in $\AA$ ), c) the radial velocity of the peaks (in $\mathrm{km} \mathrm{s}^{-1}$ ) and $\mathbf{d}$ ) the peak separation (in $\mathrm{km} \mathrm{s}^{-1}$ ). Same symbols used as in Fig. 7.

Rivinius et al. (1998) suggest that these could result from the superposition of several non-radial pulsation modes. For binary systems the presence of a companion is likely to have a significant role in the evolution of a viscous circumstellar disc, truncating it and hence preventing a steady state disc from forming - possibly leading to instabilities that could result in disc loss (Negueruela et al. 2001; Negueruela \& Okazaki 2001).

The data set presented allows us to investigate the long term evolution of the circumstellar environment of $\mathrm{X}$ Per, with the two emission lines and $V$ band photometry providing complementary diagnostics for the structure of the disc. The $V$ band continuum emission is likely to arise within the inner $2-3 R_{*}$ of the circumstellar disc and provides a measure of the base density of the disc. Telting et al. (1998) modeled the $U-L$ band photometry of X Per between 1975-1995 with the Curve of Growth prescription of Waters (1986) and found the range of $V$ band magnitudes considered $(V \sim 6.2-6.8)$ corresponded to changes in the base density of the disc by a factor of $\sim 20$ between optical maximum and minimum $\left(\rho_{0}=1.5 \pm 0.3 \times 10^{-10} \mathrm{gcm}^{-3}\right.$ for $\left.V=6.25\right)$. The origin of He I $6678 \AA$ is less certain. Stee (1998) suggests that it is likely to be formed within the inner few stellar radii of the disc. Comparing both line profiles during Phases 3 and 4 we find the typical peak separation of $\mathrm{H} \alpha$ to be 70 per cent of that for He I $6678 \AA$. Assuming a quasiKeplerian disc this suggests that the radial extent of the He I $6678 \AA$ emission region is roughly half that of $\mathrm{H} \alpha$.
Given that $\mathrm{H} \alpha$ is intrinsically a much stronger line this result is not unexpected as it originates from a more radially extended regions of the disc (e.g. Stee 1998; Marlborough et al. 1997).

It is clear from Fig. 7 that the behaviour of $\mathrm{H} \alpha$ and the $V$ band continuum is rather complicated and that a simple correlation between photometric magnitude and $\mathrm{H} \alpha$ equivalent width $(E W)$ is not present. In Fig. 9 we summarise this behaviour by plotting the $\mathrm{H} \alpha E W$ against $V$ magnitude (panel $a$ showing Phases 1 and 2, panel $b$ Phases 3 and 4). In order to follow the evolution of these physical quantities it is also useful to consider the peak separation of the $\mathrm{H} \alpha$ profile which will reflect the bulk motion of the material in the outer disc. Under the assumption of a quasi-Kelperian disc - appropriate for the disc radii considered here (Okazaki 2001) - this will provide a measure of the radius of the emitting region. Although the $\mathrm{H} \alpha$ emitting region is unlikely to ionisationally bound, we cannot a priori assume that the peak separation of $\mathrm{H} \alpha$ is due to a sharp density boundary (i.e. the "edge" of the circumstellar disc); it is more likely that it reflects the radius beyond which the disc density is too low to contribute significant line emission, which may or may not be due to a "discontinuous" change in density associated with a disc "outer edge".

During the disc loss in phase 1 (open circles) both line and continuum emission are observed to decrease, with line emission still present in $\mathrm{H} \alpha$ when the disc densities are too low to result in detectable excess optical continuum flux from the circumstellar disc. The sole source of continuum emission at this time being the stellar photosphere - resulting in the vertical evolutionary track in Fig. 9 which terminates at the point at which a purely photospheric profile is observed and no contribution from the circumstellar disc is present.

Initially the data for Phase 2 (filled circles) follows the same vertical track as seen for the final stages of disc loss in Phase 1 - clearly as the disc begins to reform it has a rather low density. While it was not possible for Telting et al. (1998) to determine the disc density gradient at this time, from the linear decrease in the $\mathrm{H} \alpha$ peak separation at this time it appears that the outer edge of the emitting region is increasing at this time. Assuming an inclination, $i=30^{\circ}$, and a quasi-Keplerian disc the radius of the $\mathrm{H} \alpha$ emitting region grows from $1.1 R_{*}-8.6 R_{*}$ in the $\sim 2000$ days from TJD 48554 (note that the intial peak separation of both $\mathrm{H} \alpha$ and He I $6678 \AA$ is greater than twice the projected rotational velocity of $\mathrm{X}$ Per, clearly indicating the transfer of angular momentum to the disc material). We note that this disc growth time is comparable to the viscous timescale at $r \sim 10 R_{*}$. The viscous timescale $\tau_{\text {vis }}$ is given by $\tau_{\text {vis }} \sim\left[\alpha(H / r)^{2} \Omega\right]^{-1}$, where $\alpha$, $H$, and $\Omega$ are the Shakura-Sunyaev's viscosity parameter, the disc scale-height, and the angular frequency of disc rotation. Adopting the stellar mass and radius on Table 1 and $T_{\text {eff }}=31000 \mathrm{~K}$ (Telting et al. 1998) and assuming that the disc is isothermal at $T_{\mathrm{d}}=\frac{1}{2} T_{\text {eff }}$, we have 

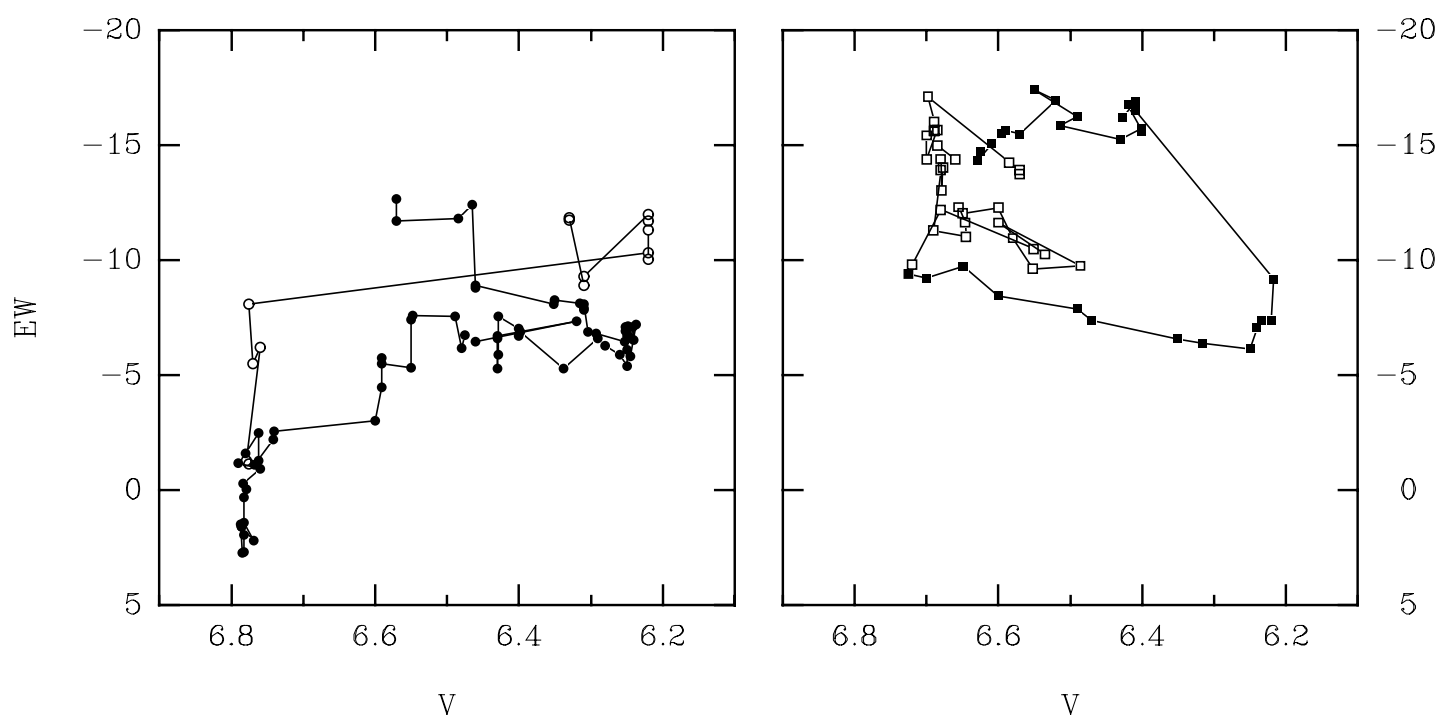

Fig. 9. Plots of the evolution of $\mathrm{H} \alpha$ Equivalent Width $(E W)$ against $V$ band magnitude for the period 1987-2000 for the 4 phases of the lightcurve as defined in Sect. 3.1. Panel 1 (left) plots data taken during phases 1 (open circles) and 2 (filled circles). Panel 2 (right) plots data from phases 3 (open squares) and 4 (filled squares).

$\tau_{\text {vis }} \sim 1.3 \alpha^{-1}\left(r / 8.6 R_{*}\right)^{1 / 2}$ yr for $\mathrm{X}$ Per. Thus, the viscous timescale at $r=8.6 R_{*}$ for $\alpha \sim 0.2$ gives the similar timescale to that observed.

Following the vertical evolution of the points in panel 1 (Fig. 9), the density in the disc becomes sufficiently large for continuum emission to be apparent and the increase in line flux is matched by the higher continuum, resulting in a $\sim$ constant $E W_{\mathrm{H} \alpha}$ until optical maximum. After this event the $V$ band continuum is observed to decrease. Given that the continuum emission is likely to be optically thin at this wavelength, this likely represents a decrease in the density (and hence emission measure) of the disc. During this time the $E W_{\mathrm{H} \alpha}$ is observed to increase as a result of the lowering continuum, suggesting a constant line flux. Given that the density in the disc is observed to decrease at this time this suggests that either the disc is at least partially optically thick to $\mathrm{H} \alpha$ (and hence the flux is proportional to the (constant) projected disc area) or that the material is preferentially lost at the inner edge of the disc (which is responsible for the continuum emission) and not at the larger radii responsible for $\mathrm{H} \alpha$ line emission.

During this period the He I line profile showed significant variability (in addition to the cyclic $V / R$ variability also present in $\mathrm{H} \alpha$; Sect. 4.2), with the appearance of a 4 peaked line profile. Once again the new peaks were observed to appear at projected velocities clearly in excess of the stellar rotational velocity. As with the original peaks, the outer peaks are also observed to migrate to line centre (e.g. Fig. 8) suggesting the drift of the emitting material to larger radii, although we note that for this process appears to occur more rapidly than for the first set of peaks.

Tarasov \& Roche (1995) suggest this results from a nested double disc structure - however it is not clear that there is a region devoid of material in the equatorial plane, separating an inner disc from an annulus at larger radii. What appears more likely is that the density of material in the equatorial plane has increased at very small radii at this time and consequently the radial density gradient of the disc is not represented by a simple power law prescription (although we note that this is hard to reconcile given the reduction in strength of the optical continuum at this time which is also thought to arise at small radii). In this case, the migration of the second set of peaks toward the line centre results from the change in the radial density distribution toward a simple power law. This process is likely to occur more rapidly than the disc formation itself, which is traced by the change in the separation of the first set of peaks.

This process continues throughout Phase 3, where it appears that the radial extent of the $\mathrm{H} \alpha$ emitting region of the disc remains constant. We note again that this does not imply that the outer edge of the disc has ceased to expand, rather that material outside this radius is too diffuse to contribute significantly to line emission (likewise material will continue to drift outwards through this region of the disc). Both the $V$ band flux and $E W_{\mathrm{H} \alpha}$ show small variations during this time implying changes in the structure of the disc. Given the reduction in $E W_{\mathrm{H} \alpha}$ at constant photometric brightness (indicating a real reduction in line flux), followed by a short lived increase (and subsequent decrease) in photometric magnitude at constant $E W_{\mathrm{H} \alpha}$ (Fig. 9; indicating changes in both line and continuum emission) it would appear that a reduction in the emissivity of the $\mathrm{H} \alpha$ region (outer disc) can occur independently of the inner disc (responsible for the continuum). Therefore the evolution of the circumstellar disc cannot be understood as simply due to changes in the base disc density, but must also involve variations of the radial density gradient of the disc.

In a viscous decretion disc, the density distribution is expected to evolve as follows. In an early stage of disc formation, the density gradient is not a simple power law 
but is much steeper in the outer region than that in a steady disc (which has a spectral index of -2 according to Porter 1999 and Okazaki 2001). As the disc develops, the density gradient will asymptotically approach that seen in the steady disc if the mass is continually supplied from the star. If the mass supply from the star stops at some point, as we have seen in X Per, the disc begins to accrete. Since the viscous timescale is an increasing function of radius, the mass accretion onto the star preferentially occurs in an inner region. As a result, the density gradient will become flatter than that in the steady disc in the region where the mass is accreting. The radius of this region increases with time. Outside the accreting region, the density gradient will be steeper than, but asymptotically approach, that seen in the steady disc.

Throughout phase 3 we note that large amplitude $V / R$ variability is present in the line profiles, indicating the presence of an $m=1$ density wave within the disc (see Sect. 4.2).

Finally phase 4 covers the final rapid photometric brightening, plateau and fade, presumably due to the sudden addition and subsequent loss of material to the disc. Once again the behaviour of $E W_{\mathrm{H} \alpha}$ can be understood in terms of a variable continuum, the constant $E W_{\mathrm{H} \alpha}$ during the brightening suggests an increase in line flux that is proportional to the increase in continuum emission, while the increase in $E W_{\mathrm{H} \alpha}$ during the subsequent fade mirrors the behaviour seen in Phase 2, with the flux in $\mathrm{H} \alpha$ initial remaining constant despite the reduction in the continuum (leading to an increase in $E W_{\mathrm{H} \alpha}$ ) followed by a reduction in both line flux and continuum (leading to constant $E W_{\mathrm{H} \alpha}$ ). During this period, the behaviour of He I $6678 \AA$ mirrors that seen in the phase 2 with a weakening of the line profile as the star brightens with the photospheric profile becoming visible. This is followed by the appearance of new emission at high velocities during the photospheric fading, presumably indicating the presence of a larger quantity of material close to the stellar surface. It is not clear whether this represents new disc material ejected from the star or material back towards the star after the supply of material from star to disc has been "turned off".

\section{2. $V / R$ variability and global one-armed oscillations}

Cyclic $V / R$ variability is seen in both isolated Be stars and in the mass donors in Be/X-ray binaries, with periods typically ranging from $\sim 1-10$ yrs for isolated Be stars (e.g. Okazaki 1997) and shorter periods for the mass donors in X-ray binaries ( months-few years; Negueruela et al. 1998). Kato (1983), Okazaki $(1991,1997)$ and Papaloizou et al. (1992) have proposed that these variations are due to the precession of a one-armed $(m=1)$ density wave in a near-Keplerian disc around the B star. Based on 3D radiative transfer calculations in discs with the $m=1$ perturbation pattern, Hanuschik et al. (1995) and Hummel \& Hanuschik (1997) showed that the one-armed oscillation model agrees well with the observed, long-term line profile valiabilities. The $m=1$ mode is considered to be excited by a dynamical process of viscosity, $\eta$-mechanism according to Kato (2001). In general, inertial-acoustic oscillations with no node in the vertical direction, such as the $m=1$ modes in Be discs, are overstable in viscous, near-Keplerian discs (Kato 1978; Kato 2001 for general discussion of viscous excitation of disc oscillations; see also Negueruela et al. 2001 for Be stars).

As mentioned in Sect. 2 transient $V / R$ variability has been reported for X Per throughout the period between 1913-1982, and we confirm that this trend has been present in $\mathrm{H} \alpha$ during the period of our observations commencing $\sim 1000$ days after emission in $\mathrm{H} \alpha$ was first observed (we suspect that similar variability is also present in the He I profiles). The cyclic $V / R$ variability has been present in all subsequent spectra, with the amplitude of variability increasing throughout this period ( $\sim 2300$ days).

It is interesting to see even in a qualitative level whether the $m=1$ eigenmode in the disc of $\mathrm{X}$ Per has characteristics consistent with the observed features described previously. For this purpose, we construct an unperturbed disc model, solve the equations for the $m=1$ perturbation, and calculate emission line profiles from the disc with the $m=1$ perturbation pattern, by a simplified treatment described below. We assume that the unperturbed disc is steady, axisymmetric, and isothermal with temperature of $\frac{1}{2} T_{\text {eff. }}$. By the reason discussed in Sect 4.3, we assume that the unperturbed disc is truncated at the 3:1 resonance radius, where the disc particle rotates three times as fast as the frequency of mean binary rotation, but neglect the other effect of the compact companion on the disc structure. Moreover, although the mechanism of confinement of the $m=1$ waves in discs around early Be stars is not well understood, we assume that it is due to the optically-thin line force in the form $F_{\text {rad }}=\eta\left(r / R_{*}\right)^{\epsilon} \times G M_{*} / r^{2}$ (Chen \& Marlborough 1994). According to Papaloizou et al. (1992), we include the quadrupole contribution to the gravitational potential around the rotationally-distorted star, adopting $k_{2}=$ $6 \times 10^{-3}$, where $k_{2}$ is the apsidal motion constant. We adopt Shakura-Sunyaev's viscosity prescription. We use the cylindrical coordinates centred on the Be star primary.

First, we construct the unperturbed disc model. Given the radial velocity component at the stellar surface, the structure of the unperturbed disc is obtained by solving the equations describing the mass, momentum, and angular momentum conservation (see Okazaki 2001 for details). An example of such a solution is presented in Fig. 10 for $\alpha=0.2$ and $V_{\mathrm{r}}\left(R_{*}\right) / c_{\mathrm{s}}=10^{-4}$, where $V_{\mathrm{r}}$ is the radial velocity and $c_{\mathrm{s}}$ is the isothermal sound speed. The solid, dashed, and dash-dotted lines denote $\sigma / \sigma\left(R_{*}\right), V_{\mathrm{r}} / c_{\mathrm{s}}$, and $V_{\phi} / V_{\mathrm{K}}\left(R_{*}\right)$, respectively, where $\sigma$ is the surface density, $V_{\phi}$ are the azimuthal velocity, and $V_{\mathrm{K}}\left(R_{*}\right)$ is the Keplerian velocity at the stellar surface. Note that $V_{\phi} \sim r^{-1 / 2}, V_{\mathrm{r}} \sim r$, and $\sigma \sim r^{-2}$. 


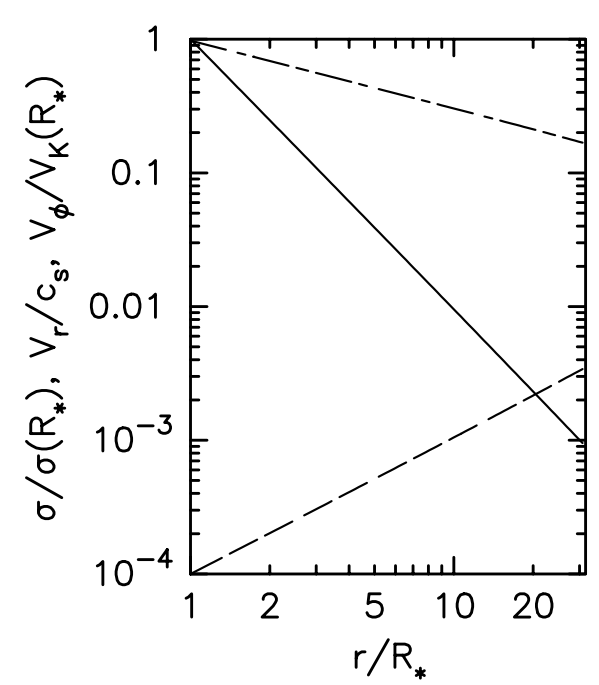

Fig. 10. Structure of the viscous decretion disc with $\alpha=$ 0.2 and $V_{\mathrm{r}}\left(R_{*}\right) / c_{\mathrm{S}}=10^{-4}$. The radiative force in the form of $\eta\left(r / R_{*}\right)^{\epsilon} \times G M_{*} / r^{2}$ with $(\eta, \epsilon)=(0.05,0.1)$ is included. According to Papaloizou et al. (1992), the quadrupole contribution to the potential around the rotationally-distorted star with $k_{2}=6 \times 10^{-3}$ is taken into account, where $k_{2}$ is the apsidal motion constant. The solid, dashed, and dash-dotted lines denote $\sigma / \sigma\left(R_{*}\right), V_{\mathrm{r}} / c_{\mathrm{s}}$, and $V_{\phi} / V_{\mathrm{K}}\left(R_{*}\right)$, respectively, where $\sigma$ is the surface density, $V_{\mathrm{r}}$ and $V_{\phi}$ are the radial and azimuthal velocity components, respectively, and $V_{\mathrm{K}}$ is the Keplerian velocity.

Next, we consider a linear, isothermal perturbation on the unperturbed disc shown in Fig. 10. Our linearised equations for the $m=1$ isothermal perturbations are the same as those used by Negueruela et al. (2001) [their Eqs. (5)-(7)]. As boundary conditions, we impose that the horizontal velocity perturbation vanishes at the inner edge of the disc and $\Delta p=0$ at the outer disc radius, where $\Delta p$ is the Lagrangian perturbation of pressure. Solving those equations with the boundary conditions, we have the fundamental $m=1$ eigenmode shown in Fig. 11. The mode, which is confined to $r \lesssim 10 R_{*}$ and precesses in a prograde manner, has a period of $2.5 \mathrm{yr}$ and the growth time of $5.7 \mathrm{yr}$, both of which roughly agrees with the observed quasi-periodicity and the growth timescale of $V / R$ variation in the $\mathrm{H} \alpha$ line. Given that the growth time is proportional to $\alpha^{-1}$ (Negueruela et al. 2001), this agreement implies that the viscosity parameter $\alpha$ in the disc of $\mathrm{X}$ Per cannot be much bigger or smaller than 0.2. Recall that the same conclusion has been obtained from the comparison between the disc growth timescale and the viscous timescale (Sect 4.1).

As mentioned in Sect 3.1, the cycle length of the $\mathrm{H} \alpha$ line variability has decreased from $\sim 2.0 \mathrm{yr}$ to $\sim 0.6 \mathrm{yr}$ over three cycles in Phase 3. During this period, the $E W$ of the $\mathrm{H} \alpha$ line was gradually decreasing and the $V$ band magnitude was fluctuating at a low level (see Fig. 7), suggesting the disc structure was changing during this period. Since the $m=1$ mode is more easily confined and has a higher eigenfrequency in a disc with a flatter density distribution (e.g., Okazaki 1997), we suspect that the accretion of the inner region of the disc caused a flattening in the radial density distribution during this period.

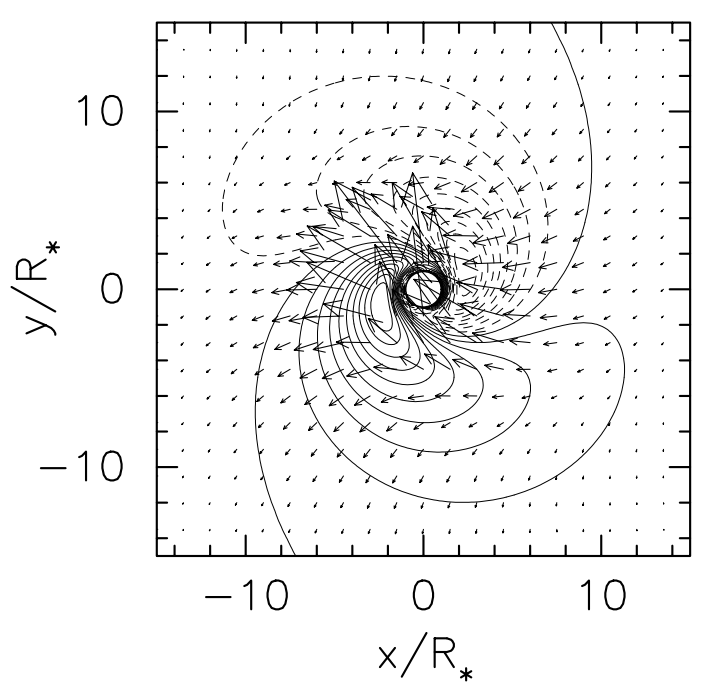

Fig. 11. Linear, isothermal $m=1$ mode in the viscous disc given in Fig. 9. Both disc and mode rotate counterclockwise. The contours denote the relative density perturbation in linear scale. The solid (dashed) contours are for the region with positive (negative) density enhancement. Arrows superposed on the contours denote the perturbed velocity vectors normalized by the unperturbed azimuthal velocity $V_{\phi}$. The disc outer radius is at $r \simeq 30.8 R_{*}$.

Finally, we calculate the emission line profiles, assuming that the nonlinear perturbation pattern is similar to that of the linear perturbation shown in Fig. 11. In order to show how large the amplitude of the observed $V / R$ variation is, we adopt an extremely large amplitude, $90 \%$, of relative density perturbation and compare the resulting profiles with the observed ones. We compute the Balmer-line profiles using the same method as described in Okazaki (1996), but now we include the stellar continuum and the deviation from the second energy level of hydrogen. We assume that the stellar source function is equal to the Planck function at $T_{\text {eff }}$ and that the deviation factor, $b_{2}$, is given by $b_{2}=1 / W$, where $W$ is the dilution factor defined by $W=\frac{1}{2}\left\{1-\left[1-\left(R_{*} / r\right)^{2}\right]^{1 / 2}\right\}$ (e.g., Hirata \& Kogure 1984).

Figure 12 shows model Balmer-line profiles for $\tau_{0}=$ $10^{3}$, where $\tau_{0}$ is the line optical depth from the unperturbed disc when it is seen pole-on. The inclination angle $i$ is $30^{\circ}$ and the velocity resolution of each profile is $10 \mathrm{~km} \mathrm{~s}^{-1}$ (the resolving power is about 30000 for the $\mathrm{H} \alpha$ line), which is about the same as the spectra presented in Figs. 1-6. In the figure, the variabilities due to the $m=1$ perturbation pattern is shown by profiles at eight different phases.

From Fig. 12, we note that the line-profile variability due to the $m=1$ mode qualitatively agrees with the observed $V / R$ variations in the $\mathrm{H} \alpha$ line. The profile as a whole shifts blueward (redward) when the red (blue) peak is the stronger, as seen in many Be stars. Therefore, it is likely that the observed $V / R$ variation in the $\mathrm{H} \alpha$ line in $\mathrm{X}$ Per is due to an overstable $m=1$ mode in a viscous decretion disc. As the mode grows, the amplitude of the 


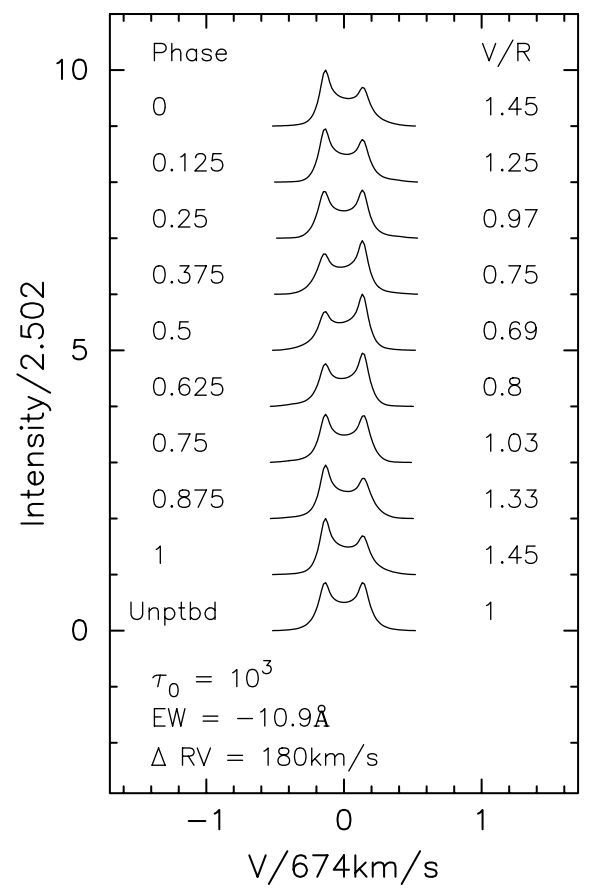

Fig. 12. Variabilities in the Balmer-line profiles due to the $m=$ 1 perturbation pattern for $\tau_{0}=10^{3}$. The line-profile variability is shown by the profiles at eight different phases. The profile labeled "Unptbd" is for the unperturbed disc. The intensity is normalized by the continuum level. On both sides of each profile, the phase and the $V / R$ ratio are given. At phase 0 , the observer is at the $x$-direction of Fig. 10. The equivalent width and the peak separation of the unperturbed profile are given at the bottom of the figure.

$V / R$ variation increases. It is important to note that the increase in the amplitude of the $V / R$ variations in viscous decretion discs is expected to occur gradually (and exponentially), taking years to a decade, because of a long growth time of the mode. This is exactly what we have found in the $V / R$ variation in X Per (see Fig. 7c).

A closer look at the model profile, however, reveals that the agreement is quantitatively not good at replicating some physical parameters. Despite the fact that we have adopted an extremely large amplitude of perturbation, the amplitude of the observed $V / R$ variation in the $\mathrm{H} \alpha$ line is still larger than that of the model profile. The amplitude in the velocity shift of the peaks is also larger in the observed $\mathrm{H} \alpha$ profiles $\left(\sim 10-20 \mathrm{~km} \mathrm{~s}^{-1}\right)$ than in the model profiles $\left(<10 \mathrm{kms}^{-1}\right.$ for $\left.\tau_{0}=10^{3}\right)$. We take this discrepancy in a quantitative level as evidence that the $m=1$ mode in the disc of X Per has been highly nonlinear throughout Phases 3 and 4 , where the linear $m=1$ perturbation does not fit well. To fully understand the long-term behaviour of the disc of X Per, we need to perform a nonlinear simulation of the evolution of a viscous decretion disc, which is beyond the scope of this paper.

\subsection{Interaction with the compact companion}

Since X Per has a low orbital eccentricity $(e=0.11)$ and a long orbital period $\left(P_{\text {orb }}=250 \mathrm{~d}\right)$ and the mass ratio $q$ between the neutron star companion and the Be star primary is low $(q=0.09)$, in the previous subsection we have safely neglected the effect of the neutron star on the structure of an inner part of the disc, to which the $m=1$ mode is confined. However, the neutron star can affect the disc structure in an outer region. Below we discuss the long-term effect of the neutron star on the disc structure.

Recently, Negueruela \& Okazaki (2001) and Okazaki \& Negueruela (2001) studied the interaction of the Be disc and the neutron star in Be/X-ray binaries by a semianalytical method, and found that the $\mathrm{Be}$ disc in $\mathrm{Be} / \mathrm{X}$ ray binaries is truncated through the resonant interaction with the neutron star and that the truncation radius depends mainly on the orbital parameters and the viscosity. Although the orbital eccentricity of seven systems to which they applied their model ranged between 0.31 and 0.88, Okazaki \& Negueruela (2001) also pointed out that the Be disc in systems with very low orbital eccentricity is expected to be truncated at the $3: 1$ resonance radius not by the resonant interaction but by the tidally-driven eccentric instability, the same mechanism that truncates accretion discs in circular binaries with $0.05 \lesssim q \lesssim 0.25$ (e.g., Osaki 1996).

The implicit assumption they made is that, in systems with very-low eccentricity, the resonant torque is negligible compared to the viscous torque inside the $3: 1$ radius. This always holds for circular binaries, where the resonant torque becomes non-zero only at the 2:1 resonance radius. For $e \neq 0$, however, this assumption is not trivial even if $e \ll 1$. Therefore, below we apply their model to X Per to test this assumption.

In the model presented by Negueruela \& Okazaki (2001) the viscous torque is evaluated by the formula derived by Lin \& Papaloizou (1986), while the resonant torque is calculated at each resonance radius by using the torque formula given by Goldreich \& Tremaine $(1979,1980)$ after decomposing the binary potential into a Fourier series (see Negueruela \& Okazaki 2001 for details). The criterion for the disc truncation at a given resonance radius is that the viscous torque is smaller than the resonant torque at that radius. This criterion is met for $\alpha$ smaller than a critical value, $\alpha_{\text {crit }}$. In Fig. 13 , we plot $\alpha_{\text {crit }}$ at several $n: 1$ resonance radii. The resonant torques at the $n: 1$ radii are stronger than those at radii with other period commensurabilities located nearby. The parameters adopted are the same as those adopted for modelling the unperturbed disc and the $m=1$ mode in the previous subsection, except that $\alpha$ is now a free parameter.

Figure 13 shows that if the non-resonant torque does not work, the disc of X Per will be truncated at the 2:1 or $3: 1$ resonance radius, depending on whether $2.3 \times 10^{-2} \lesssim$ $\alpha \lesssim 0.66$ or $6.8 \times 10^{-4} \lesssim \alpha \lesssim 2.3 \times 10^{-2}$, respectively, and will not be truncated for $\alpha \gtrsim 0.66$. At the $3: 1$ radius, however, the non-resonant tidal torque, which is responsible for the tidally-driven eccentric instability, is likely to dominate the viscous torque irrespective of $\alpha$. Consequently, we expect that the disc of X Per is truncated at the 3:1 radius for a plausible range of $\alpha\left(\alpha>7 \times 10^{-4}\right)$, as expected by Okazaki \& Negueruela (2001). 


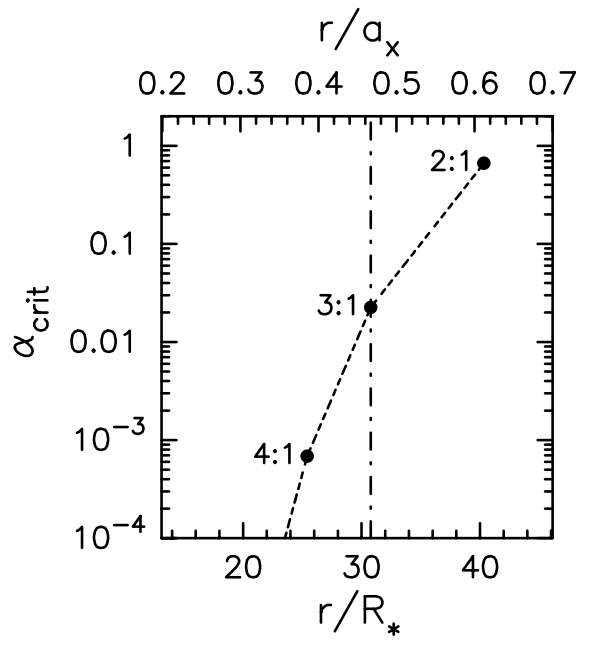

Fig. 13. Critical values of $\alpha$ at some resonance radii for $T_{\mathrm{d}}=$ $\frac{1}{2} T_{\text {eff }}$. For other disc temperature, $\alpha_{\text {crit }}$ should be multiplied by a factor of $T_{\text {eff }} / 2 T_{\mathrm{d}}$. Annotated in the figure are the locations of the $n: 1$ commensurabilities of disc and mean binary orbital frequencies. The vertical line denotes the radius, at which the non-resonant tidal torque is expected to truncate the disc even if $\alpha \gg \alpha_{\text {crit }}$. The radius $r / R_{*}$ is calculated for $i=30^{\circ}$.

X Per appears to be one of a small group of Be X-ray binaries which are low luminosity persistent sources with small increases in X-ray luminosity even during "outburst". (e.g. RX J0440.9+4431 and RX J1037.5-564; Reig \& Roche 1999). X-ray monitoring of X Per during the period of these observations confirmed this trend; no major X-ray outbursts or variability was observed throughout this time. This behaviour can naturally be explained by the truncation of the decretion disc by the neutron star companion. Truncation at the 3:1 resonance radius makes a wide gap between the disc outer radius $\left(0.47 a_{x}\right)$ and the periastron distance $\left(0.89 a_{x}\right)$. This wide gap will result in disc truncation in this system being so effective that the neutron star hardly accretes any gas from the Be disc even at periastron - with the disc acccumulating most of the material ejected from the star - naturally leading to a low X-ray luminosity.

In the viscous decretion disc model, which we have shown naturally explains many observed features of X Per, the disc forms in a viscous timescale $\tau_{\text {vis. }}$. Given that $\tau_{\text {vis }} \sim\left[\alpha(H / r)^{2} \Omega\right]^{-1} \sim 2.5 \alpha^{-1}\left(r / 0.47 a_{x}\right)^{1 / 2}$ yr for parameters for X Per, the disc truncation is expected to begin to work at $\sim 12(\alpha / 0.2)^{-1} \mathrm{yr}$ after the disc formation begins. Before this time, the disc evolution will be no different from that in isolated Be stars, but once the disc truncation begins to work, the density distribution will increase with time, making the radial density distribution flatter than that in the disc around isolated Be stars. As for our photometric and spectroscopic observations during the period of 1987-2000, only the very recent observations could be affected by the disc truncation if $\alpha \sim 0.2$. Future observations will reveal interesting features in the evolution of the truncated disc if the Be star continues to supply material to the disc.

\section{Conclusions}

We have presented the results of a 13 year spectroscopic and photometric monitoring campaign for X Persei which show that throughout this period the star showed significant variability which we attribute to changes in the mass content and distribution in the circumstellar disc. Our data show that there was one episode of complete disc loss during this period which was quickly followed by disc reformation. The data also show the formation and growth of a perturbation to the disc which we attribute to the presence of a one armed density wave. Lack of correlation between the strength of the line and continuum emission demonstrate that disc variability involves (at least in part) a redistribution of material within the disc, leading to changes in the radial density gradient, rather than changes in the base density of the disc with a constant density gradient. We interpret these observations semi-analytically as variations in a viscous, quasi-Keplerian decretion disc around the Be star primary. From the timescale of disc growth we find a value for $\alpha$, the Shakura-Sunyaev viscosity parameter, of $\sim 0.2$. Adopting this value for the simulation of the growth and periodicity of a one armed density wave in an isothermal Keplerian disc we find a timescale for growth of $\sim 5.7$ yrs and a periodicity of $\sim 2.5$ yrs, encouragingly close to the observations. Changes in the periodicity of this wave can further be ascribed to a flattening of the density gradient of the inner regions of the disc, qualitatively consistent with the expected behaviour of a quasi-Keplerian disc if the source of angular momentum at the star/disc boundary is "switched off" (note that we cannot provide a mechanism for such transport of material and angular momentum from star to disc). Finally, the value of $\alpha=0.2$ is also consistent with the truncation of the circumstellar disc well within the orbital radius of the neutron star companion, effectively preventing the transfer of material onto the neutron star, explaining the observed low X-ray flux and lack of significant flaring activity. We conclude that the viscous decretion disc theory for Be star discs explains, in at least a qualitive manner, the observed properties of X Persei.

Acknowledgements. JSC gratefully acknowledges PPARC funding. AET gratefully acknowlege receipt of financial support from the Royal Society for collaborative work with the Former Soviet Union. We also thank Dr. V. Simon for providing valuable additional spectroscopic observations.

\section{Appendix A: Observational details}

Here we list the basic parameters of the spectral data utilised in this paper.

Table A.1 summarises the $\mathrm{H} \alpha$ observations covering 1987 to 1998 - the source of each spectrum corresponds to the key given in Sect. 2.

Table A.2 summarises the HeI $\lambda 6678$ observations covering 1991 to 1998 - the source of each spectrum corresponds to the key given in Sect. 2. 
Table A.1. H $\alpha$ observations of X Persei. TJD $=$ JD -2400000 .

\begin{tabular}{|c|c|c|c|c|c|c|c|c|c|c|c|}
\hline TJD & & $\begin{array}{l}E W \\
(\AA)\end{array}$ & $V / R$ & TJD & & $\begin{array}{l}E W \\
(\AA)\end{array}$ & $V / R$ & $\begin{array}{l}\text { TJD } \\
(\AA)\end{array}$ & & $E W$ & $V / R$ \\
\hline 47065.57 & $\mathrm{cr}$ & -11.75 & 1.64 & 49641.59 & $\mathrm{uk}$ & -6.69 & 1.03 & 51172.18 & $\mathrm{cr}$ & -8.45 & 0.87 \\
\hline 47066.50 & $\mathrm{cr}$ & -11.86 & 1.59 & 49642.66 & fr & -7.14 & 1.01 & 51186.27 & $\mathrm{cr}$ & -7.89 & 0.95 \\
\hline 47153.8 & uk & -8.9 & - & 49642.59 & uk & -6.52 & 1.03 & 51192.41 & $\mathrm{cr}$ & -7.37 & 0.99 \\
\hline 47160.7 & uk & -9.3 & - & 49643.62 & fr & -7.12 & 1.01 & 51221.41 & $\mathrm{cr}$ & -6.58 & 1.15 \\
\hline 47229.7 & uk & -12.0 & - & 49645.60 & $\mathrm{cz}$ & -6.61 & 1.05 & 51235.24 & $\mathrm{cr}$ & -6.37 & 1.22 \\
\hline 47230.6 & $\mathrm{uk}$ & -11.7 & - & 49646.64 & $\mathrm{cz}$ & -6.45 & 1.04 & 51381.52 & $\mathrm{cr}$ & -7.07 & 0.87 \\
\hline 47231.7 & $\mathrm{uk}$ & -11.3 & - & 49649.77 & $\mathrm{uk}$ & -6.83 & 1.04 & 51381.52 & $\mathrm{cr}$ & -7.07 & 0.87 \\
\hline 47235.28 & $\mathrm{cr}$ & -10.05 & 1.36 & 49654.35 & $\mathrm{cr}$ & -6.90 & 1.11 & 51393.55 & $\mathrm{cr}$ & -7.39 & 0.85 \\
\hline 47237.28 & $\mathrm{cr}$ & -10.33 & 1.33 & 49672.27 & $\mathrm{cr}$ & -8.10 & 1.06 & 51422.54 & $\mathrm{cr}$ & -7.38 & 0.83 \\
\hline 47925.6 & uk & -1.3 & - & 49687.54 & $\mathrm{cr}$ & -7.92 & 0.99 & 51475.30 & $\mathrm{cr}$ & -9.18 & 0.84 \\
\hline 47950.4 & $\mathrm{uk}$ & -1.14 & 1.03 & 49689.52 & $\mathrm{cr}$ & -7.85 & 1.09 & 51769.48 & $\mathrm{cr}$ & -16.52 & 1.60 \\
\hline 48137.6 & $\mathrm{uk}$ & 1.6 & 1.00 & 49692.46 & $\mathrm{cz}$ & -8.12 & 1.00 & 51771.44 & $\mathrm{cr}$ & -16.89 & 1.56 \\
\hline 48194.55 & fr & 2.71 & - & 49692.62 & $\mathrm{cr}$ & -8.28 & 1.12 & 51777.53 & $\mathrm{cr}$ & -16.22 & 1.57 \\
\hline 48211.7 & $\mathrm{uk}$ & 2.7 & - & 49694.45 & $\mathrm{cz}$ & -8.09 & 1.01 & 51801.44 & $\mathrm{cr}$ & -16.74 & 1.67 \\
\hline 48253.5 & $\mathrm{uk}$ & 1.93 & - & 49705.30 & $\mathrm{cz}$ & -8.92 & 1.01 & 51825.42 & $\mathrm{cr}$ & -15.60 & 1.65 \\
\hline 48284.3 & $\mathrm{uk}$ & 2.2 & - & 49708.41 & $\mathrm{cr}$ & -8.80 & 1.02 & 51858.18 & $\mathrm{cr}$ & -15.74 & 1.50 \\
\hline 48497.3 & uk & 1.4 & 1.00 & 49761.4 & $\mathrm{cr}$ & -11.81 & - & 51865.24 & $\mathrm{cr}$ & -15.26 & 1.64 \\
\hline 48554.68 & fr & 0.31 & 1.00 & 49762.37 & $\mathrm{cr}$ & -11.7 & 0.93 & 51887.27 & $\mathrm{cr}$ & -15.86 & 1.63 \\
\hline 48557.3 & $\mathrm{uk}$ & -0.05 & 1.01 & 49778.29 & $\mathrm{cz}$ & -12.67 & 0.94 & 51892.28 & $\mathrm{cr}$ & -16.25 & 1.64 \\
\hline 48671.3 & $\mathrm{uk}$ & -0.93 & 1.00 & 49934.74 & uk & -13.90 & - & 51917.37 & $\mathrm{cr}$ & -17.43 & 1.52 \\
\hline 48851.3 & $\mathrm{uk}$ & -1.18 & 0.96 & 49936.71 & uk & -13.59 & - & 51952.19 & $\mathrm{cr}$ & -16.94 & 1.54 \\
\hline 48852.3 & $\mathrm{uk}$ & $-2.91:$ & 0.94 & 49937.74 & uk & -13.91 & - & 52027.28 & $\mathrm{cr}$ & -15.48 & 1.23 \\
\hline 48932.56 & fr & -1.600 & 0.99 & 49937.53 & $\mathrm{cr}$ & -13.73 & 1.40 & 52092.53 & $\mathrm{cr}$ & -15.63 & 1.18 \\
\hline 48940.3 & $\mathrm{uk}$ & -2.50 & 0.99 & 49946.55 & $\mathrm{cr}$ & -14.24 & 1.45 & 52097.53 & $\mathrm{cr}$ & -15.52 & 1.14 \\
\hline 49055.3 & $\mathrm{uk}$ & -2.58 & 1.02 & 49999.39 & $\mathrm{cr}$ & -17.10 & 1.37 & 52149.48 & $\mathrm{cr}$ & -15.04 & 0.99 \\
\hline 49057.3 & $\mathrm{uk}$ & -3.03 & 1.01 & 50007.60 & $\mathrm{fr}$ & -16.03 & 1.42 & 52164.51 & $\mathrm{cr}$ & -14.34 & 0.95 \\
\hline 49212.3 & $\mathrm{uk}$ & -4.46 & 1.00 & 50008.60 & fr & -15.60 & 1.39 & 52172.51 & $\mathrm{cr}$ & -14.71 & 0.90 \\
\hline 49251.66 & $\mathrm{cz}$ & -5.75 & 1.02 & 50010.58 & fr & -15.62 & 1.42 & 52177.45 & $\mathrm{cr}$ & -14.43 & 0.91 \\
\hline 49254.3 & $\mathrm{uk}$ & -5.51 & 1.01 & 50016.44 & fr & -15.64 & 1.31 & 52186.37 & $\mathrm{cr}$ & -13.51 & 0.87 \\
\hline 49255.3 & $\mathrm{uk}$ & -5.31 & 1.01 & 50051.53 & uk & -14.37 & - & & & & \\
\hline 49296.48 & $\mathrm{cr}$ & -7.40 & 1.02 & 50052.32 & $\mathrm{cr}$ & -15.42 & 1.14 & & & & \\
\hline 49297.42 & $\mathrm{cr}$ & -7.60 & 1.03 & 50070.43 & $\mathrm{cr}$ & -14.99 & 1.15 & & & & \\
\hline 49305.47 & $\mathrm{cr}$ & -7.56 & 1.01 & 50100.27 & $\mathrm{cr}$ & -14.38 & 1.16 & & & & \\
\hline 49327.4 & $\mathrm{uk}$ & -6.18 & - & 50114.29 & $\mathrm{cr}$ & -14.01 & 1.07 & & & & \\
\hline 49335.43 & $\mathrm{cr}$ & -6.73 & 0.99 & 50140.24 & $\mathrm{cr}$ & -13.02 & 0.92 & & & & \\
\hline 49341.42 & $\mathrm{cr}$ & -6.47 & 0.98 & 50142.46 & uk & -14.39 & 0.97 & & & & \\
\hline 49358.15 & $\mathrm{cr}$ & -7.35 & 0.99 & 50143.41 & uk & -13.90 & 0.95 & & & & \\
\hline 49373.43 & $\mathrm{cr}$ & -6.719 & 0.99 & 50293.54 & $\mathrm{cr}$ & -11.28 & 0.73 & & & & \\
\hline 49386.42 & $\mathrm{cr}$ & -5.28 & 0.96 & 50324.50 & $\mathrm{cr}$ & -11.02 & 0.81 & & & & \\
\hline 49400.34 & $\mathrm{cr}$ & -6.60 & 1.02 & 50360.42 & $\mathrm{cr}$ & -11.63 & 0.98 & & & & \\
\hline 49401.39 & $\mathrm{cr}$ & -5.89 & 0.99 & 50393.58 & $\mathrm{cr}$ & -12.30 & 1.16 & & & & \\
\hline 49411.4 & $\mathrm{uk}$ & -7.57 & - & 50406.30 & $\mathrm{cr}$ & -12.04 & 1.30 & & & & \\
\hline 49413.31 & $\mathrm{cr}$ & -7.01 & 0.98 & 50430.38 & $\mathrm{cr}$ & -12.28 & 1.39 & & & & \\
\hline 49437.4 & $\mathrm{uk}$ & -6.72 & - & 50466.26 & $\mathrm{cr}$ & -10.96 & 1.53 & & & & \\
\hline 49438.4 & $\mathrm{uk}$ & -6.87 & - & 50479.26 & $\mathrm{cr}$ & -9.63 & 1.47 & & & & \\
\hline 49439.4 & $\mathrm{uk}$ & -5.30 & - & 50504.42 & $\mathrm{cr}$ & -9.76 & 1.35 & & & & \\
\hline 49565.55 & $\mathrm{cr}$ & -6.60 & 1.12 & 50658.52 & $\mathrm{cr}$ & -11.63 & 0.71 & & & & \\
\hline 49587.53 & $\mathrm{cz}$ & -6.30 & 1.08 & 50730.38 & $\mathrm{cr}$ & -10.25 & 0.66 & & & & \\
\hline 49588.53 & $\mathrm{cz}$ & -5.90 & 1.06 & 50755.29 & $\mathrm{cr}$ & -10.49 & 0.68 & & & & \\
\hline 49592.47 & $\mathrm{cz}$ & -5.41 & 1.05 & 50859.19 & $\mathrm{cr}$ & -12.19 & 1.03 & & & & \\
\hline 49599.54 & $\mathrm{cz}$ & -6.12 & 1.04 & 51006.54 & $\mathrm{cr}$ & -9.81 & 1.22 & & & & \\
\hline 49600.54 & $\mathrm{cz}$ & -5.81 & 1.05 & 51052.48 & $\mathrm{cr}$ & -9.40 & 0.91 & & & & \\
\hline 49612.4 & $\mathrm{uk}$ & -7.20 & - & 51098.43 & $\mathrm{cr}$ & -9.21 & 0.83 & & & & \\
\hline 49640.57 & fr & -7.10 & 1.01 & 51115.42 & $\mathrm{cr}$ & -9.72 & 0.78 & & & & \\
\hline 49641.37 & $\mathrm{cr}$ & -6.93 & 1.04 & 51121.43 & $\mathrm{cr}$ & -9.66 & 0.78 & & & & \\
\hline 49641.61 & $\mathrm{fr}$ & -7.05 & 1.00 & 51126.42 & $\mathrm{cr}$ & -9.72 & 0.77 & & & & \\
\hline
\end{tabular}


Table A.2. HeI $\lambda 6678$ observations of X Persei.

\begin{tabular}{|c|c|c|c|c|c|c|c|c|}
\hline TJD & & $\begin{array}{l}E W \\
(\AA)\end{array}$ & TJD & & $\begin{array}{l}E W \\
(\AA)\end{array}$ & TJD & & $\begin{array}{l}E W \\
(\AA)\end{array}$ \\
\hline 48284.4 & uk & 0.85 & 49692.46 & $\mathrm{cZ}$ & -0.04 & 50859.28 & $\mathrm{cr}$ & -0.979 \\
\hline 48557.3 & uk & 0.25 & 49692.58 & $\mathrm{cr}$ & 0.064 & 51008.54 & $\mathrm{cr}$ & -0.703 \\
\hline 48671.4 & uk & 0.49 & 49694.45 & $\mathrm{cz}$ & -0.02 & 51020.46 & $\mathrm{cr}$ & -0.763 \\
\hline 48851.4 & uk & 0.07 & 49705.30 & $\mathrm{cZ}$ & -0.11 & 51094.42 & $\mathrm{cr}$ & -0.730 \\
\hline 48852.4 & uk & -0.22 & 49708.45 & $\mathrm{cr}$ & -0.111 & 51112.38 & $\mathrm{cr}$ & -0.845 \\
\hline 48940.4 & uk & -0.12 & 49761.4 & uk & -0.46 & 51126.45 & $\mathrm{cr}$ & -0.907 \\
\hline 49055.4 & uk & -0.26 & 49762.41 & $\mathrm{cr}$ & -0.339 & 51186.31 & $\mathrm{cr}$ & -0.419 \\
\hline 49057.4 & uk & -0.21 & 49934.74 & uk & -1.13 & 51192.43 & $\mathrm{cr}$ & -0.347 \\
\hline 49251.62 & $\mathrm{cz}$ & -0.25 & 49936.71 & uk & -1.20 & 51235.19 & $\mathrm{cr}$ & -0.050 \\
\hline 49254.4 & uk & -0.25 & 49937.74 & uk & -1.05 & 51285.24 & $\mathrm{cr}$ & 0.018 \\
\hline 49255.4 & uk & -0.26 & 49944.51 & $\mathrm{cr}$ & -1.342 & 51381.54 & $\mathrm{cr}$ & 0.204 \\
\hline 49296.51 & $\mathrm{cr}$ & -0.416 & 49970.48 & $\mathrm{cr}$ & -1.883 & 51396.55 & $\mathrm{cr}$ & 0.046 \\
\hline 49297.46 & $\mathrm{cr}$ & -0.482 & 49975.53 & $\mathrm{cr}$ & -1.891 & 51422.59 & $\mathrm{cr}$ & 0.081 \\
\hline 49305.51 & $\mathrm{cr}$ & -0.458 & 50006.52 & $\mathrm{cr}$ & -1.622 & 51475.36 & $\mathrm{cr}$ & -0.105 \\
\hline 49306.48 & $\mathrm{cr}$ & -0.484 & 50051.53 & uk & -1.33 & 51825.46 & $\mathrm{cr}$ & -1.121 \\
\hline 49335.46 & $\mathrm{cr}$ & -0.187 & 50052.39 & $\mathrm{cr}$ & -1.413 & 51858.22 & $\mathrm{cr}$ & -0.904 \\
\hline 49336.47 & $\mathrm{cr}$ & -0.177 & 50069.39 & $\mathrm{cr}$ & -1.522 & 51865.26 & $\mathrm{cr}$ & -0.780 \\
\hline 49341.48 & $\mathrm{cr}$ & -0.233 & 50100.23 & $\mathrm{cr}$ & -1.334 & 51892.25 & $\mathrm{cr}$ & -1.007 \\
\hline 49373.48 & $\mathrm{cr}$ & -0.083 & 50114.31 & $\mathrm{cr}$ & -1.280 & 51952.23 & $\mathrm{cr}$ & -0.995 \\
\hline 49400.35 & $\mathrm{cr}$ & -0.089 & 50139.41 & $\mathrm{cr}$ & -1.414 & 52091.53 & $\mathrm{cr}$ & -1.180 \\
\hline 49587.53 & $\mathrm{cz}$ & 0.06 & 50142.46 & uk & -1.38 & 52149.53 & $\mathrm{cr}$ & -1.132 \\
\hline 49588.53 & $\mathrm{cz}$ & 0.02 & 50143.41 & uk & -1.20 & 52164.52 & $\mathrm{cr}$ & -1.051 \\
\hline 49592.47 & $\mathrm{cz}$ & 0.10 & 50274.52 & $\mathrm{cr}$ & -0.823 & 52172.48 & $\mathrm{cr}$ & -1.079 \\
\hline 49599.54 & $\mathrm{cz}$ & 0.06 & 50293.53 & $\mathrm{cr}$ & -0.891 & 52177.48 & $\mathrm{cr}$ & -1.062 \\
\hline 49600.54 & $\mathrm{cz}$ & 0.04 & 50324.52 & $\mathrm{cr}$ & -0.875 & 52186.39 & $\mathrm{cr}$ & -0.883 \\
\hline 49641.39 & $\mathrm{cr}$ & 0.076 & 50360.45 & $\mathrm{cr}$ & -0.981 & & & \\
\hline 49641.59 & uk & 0.074 & 50392.48 & $\mathrm{cr}$ & -1.011 & & & \\
\hline 49642.64 & uk & 0.104 & 50406.28 & $\mathrm{cr}$ & -0.987 & & & \\
\hline 49645.60 & $\mathrm{uk}$ & 0.15 & 50430.40 & $\mathrm{cr}$ & -0.991 & & & \\
\hline 49646.64 & $\mathrm{uk}$ & 0.07 & 50466.27 & $\mathrm{cr}$ & -0.720 & & & \\
\hline 49649.77 & $\mathrm{uk}$ & 0.051 & 50487.39 & $\mathrm{cr}$ & -0.381 & & & \\
\hline 49654.37 & $\mathrm{cr}$ & 0.086 & 50504.41 & $\mathrm{cr}$ & -0.380 & & & \\
\hline 49672.24 & $\mathrm{cr}$ & -0.037 & 50658.53 & $\mathrm{cr}$ & -0.827 & & & \\
\hline 49687.54 & $\mathrm{cz}$ & -0.03 & 50730.39 & $\mathrm{cr}$ & -0.508 & & & \\
\hline 49689.52 & $\mathrm{cz}$ & -0.03 & 50755.26 & $\mathrm{cr}$ & -0.571 & & & \\
\hline
\end{tabular}

\section{References}

Brucato, R. J., \& Kristian, J. 1972, ApJ, 173, L105

Cowley, A. P., McLaughlin, D. B., Toney, J., \& MacConnell, D. J. 1972, PASP, 84, 834

Delgado-Marti, H., Levine, A., Pfahl, E., \& Rappaport, S. A. 2001, ApJ, 546, 455

Chen, H., \& Marlborough, J. M. 1994, ApJ, 427, 1005

Dougherty, S. M., \& Taylor, A. R. 1994, MNRAS, 269, 1123.

Fleming, W. P. 1912, Ann. Harvard Ob., 56, 165

Galkina, T. S. 1977, Izv. Crimean Obs., 57, 45

Galkina, T. S. 1980, Izv. Crimean Obs., 61, 77

Galkina, T. S. 1983, Izv. Crimean Obs., 66, 149

Galkina, T. S. 1986a, Izv. Crimean Obs., 74, 23

Galkina, T. S. 1986b, Izv. Crimean Obs., 75, 163

Goldreich, P., \& Tremaine, S. 1979, ApJ, 233, 857
Goldreich, P., \& Tremaine, S. 1980, ApJ, 241, 425

Hanuschik, R. W., Dachs, J., Baudzus, M., \& Thimm, G. 1993, A\&A, 274, 356

Hanuschik, R. W., Hummel, W., Dietle, O., \& Sutorios, E. 1995, A\&A 300, 163

Hirata, R., \& Kogure, T. 1984, Bull. Astr. Soc. India, 12, 109

Hummel, W., \& Hanuschik, R. W. 1997, A\&A, 320, 852

Kato, S. 1978, MNRAS, 185, 629

Kato, S. 1983, PASJ, 35, 249

Kato, S. 2001, PASJ, 53, 1

Kunjaya, C., \& Hirata, R. 1995, PASJ, 47, 589

Lee, U., Saio, H., \& Osaki, Y. 1991, MNRAS, 250, 432

Lin, D. N. C., \& Papaloizou, J. 1986, ApJ, 307, 395

Lyubimkov, L. S., Rostopchin, S., Roche, P., \& Tarasov, A. E. 1997, MNRAS, 286, 549 
Marlborough, J. M., Zijlstra, J.-W., \& Waters, L. B. F. M. 1997, A\&A, 321, 867

Mc Laughlin, D. B. 1937, ApJ, 85, 181

Muller, G., \& Kempf, P. 1907, Astron. Nachr., 175, 161

Negueruela, I., Reig, P., Coe, M. J., \& Fabregat, J. 1998, A\&A, 336,251

Negueruela, I., \& Okazaki, A. T. 2001, A\&A, 369, 108

Negueruela, I., Okazaki, A. T., Fabregat, J., et al. 2001, A\&A, 369,117

Norton, A. J., Coe, M. J., Estela, A., et al. 1991, MNRAS, 253, 579

Okazaki, A. T. 1991, PASJ, 43, 75

Okazaki, A. T. 1996, PASJ, 48, 305

Okazaki, A. T. 1997, A\&A, 318, 548

Okazaki, A T. 2001, PASJ, 53, 119

Okazaki, A T., \& Negueruela, I. 2001, A\&A, 377, 161

Osaki, Y. 1996, PASP, 108, 39

Papaloizou, J. C., Savonijie, G. J., \& Henrichs, H. F. 1992, A\&A, 265, L45
Porter, J. M. 1999, A\&A, 348, 512

Percy, R. 1992, JAAVSO, 21, 29

Penrod, G. D., \& Vogt, S. S. 1985, ApJ, 299, 653

Rivinius, Th., Baade, D., Stefl, S., et al. 1998, A\&A, 336, 177

Reig, P., Fabregat, J., \& Coe, M. J. 1997, A\&A, 322, 193

Reig, P., \& Roche, P. 1999, MNRAS, 306, 100.

Roche, P., Coe, M. J., Everall, C., et al. 1993, A\&A, 270, 122

Roche, P., Larionov, V., Tarasov, A. E., et al. 1997, A\&A, 322, 139

Stee, Ph. 2000, in The Be phenomenon in Early-Type stars, PASP Conf. Ser., 214, ed. M. A. Smith, H. F. Henrichs, \& J. Fabregat, 129

Tarasov, A. E., \& Roche, P. 1995, MNRAS, 276, L19

Telting, J. H., Waters, L. B. F. M., Roche, P., et al. 1998, MNRAS, 296, 785

Wackerling, L. R. 1972, PASP, 84, 827

Waters, L. B. F. M. 1986, A\&A, 162, 121 